\title{
On the accretion properties of young stellar objects in the L1615/L1616 cometary cloud cl $^{\star \star}$
}

\author{
K. Biazzo ${ }^{1}$, J. M. Alcalá ${ }^{2}$, A. Frasca ${ }^{1}$, M. Zusi², F. Getman ${ }^{2}$, E. Covino ${ }^{2}$, and D. Gandolfi ${ }^{3}$ \\ ${ }^{1}$ INAF - Osservatorio Astrofisico di Catania, via S. Sofia, 78, 95123 Catania, Italy \\ e-mail: katia.biazzo@oact.inaf.it \\ 2 INAF - Osservatorio Astronomico di Capodimonte, via Moiariello, 16, 80131 Napoli, Italy \\ ${ }^{3}$ Landessternwarte Königstuhl, Zentrum für Astronomie der Universität, Heidelberg, Königstuhl 12, 69117 Heidelberg, Germany
}

Received 21 May 2014 / Accepted 07 September 2014

\begin{abstract}
We present the results of FLAMES/UVES and FLAMES/GIRAFFE spectroscopic observations of 23 low-mass stars in the L1615/L1616 cometary cloud, complemented with FORS2 and VIMOS spectroscopy of 31 additional stars in the same cloud. L1615/L1616 is a cometary cloud in which the star formation was triggered by the impact of massive stars in the Orion OB association. From the measurements of the lithium abundance and radial velocity, we confirm the membership of our sample to the cloud. We use the equivalent widths of the $\mathrm{H} \alpha, \mathrm{H} \beta$, and the $\mathrm{He} \mathrm{I} \lambda 5876, \lambda 6678, \lambda 7065 \AA$ emission lines to calculate the accretion luminosities, $L_{\text {acc }}$, and the mass accretion rates, $\dot{M}_{\text {acc }}$. We find in L1615/L1616 a fraction of accreting objects ( $\left.\sim 30 \%\right)$, which is consistent with the typical fraction of accretors in T associations of similar age ( $\sim 3 \mathrm{Myr})$. The mass accretion rate for these stars shows a trend with the mass of the central object similar to that found for other star-forming regions, with a spread at a given mass that depends on the evolutionary model used to derive the stellar mass. Moreover, the behavior of the 2MASS/WISE colors with $\dot{M}_{\text {acc }}$ indicates that strong accretors with $\log \dot{M}_{\text {acc }} \gtrsim-8.5$ dex show large excesses in the $J H K$ s bands, as in previous studies. We also conclude that the accretion properties of the L1615/L1616 members are similar to those of young stellar objects in T associations, like Lupus.
\end{abstract}

Key words. accretion, accretion disks - techniques: spectroscopic - open clusters and associations: individual: L1615/L1616 stars: pre-main sequence - stars: low-mass

\section{Introduction}

The Lynds 1616 cloud (hereafter L1616; Lynds 1962), at a distance of about $450 \mathrm{pc}$, forms, with Lynds 1615 (hereafter L1615), a cometary-shaped cloud west of the Orion OB association $\left(\alpha \sim 5^{\mathrm{h}} 7^{\mathrm{m}}, \delta \sim-3^{\circ} 20^{\prime}\right.$; see review by Alcalá et al. 2008 , and references therein). This cometary cloud extends about $40^{\prime}$ in the sky and shows evidence of ongoing star formation activity that might have been triggered by the ultraviolet (UV) radiation coming from the massive stars in the Orion $\mathrm{OB}$ association (see Stanke et al. 2002, and references therein). In particular, recent studies led by Lee \& Chen (2007) support the validity of the radiation-driven implosion mechanism, where UV photons from luminous massive stars create expanding ionization fronts to evaporate and compress nearby clouds into bright-rimmed or comet-shaped clouds, like L1615/L1616. Implosive pressure then causes dense clumps to collapse, prompting the formation of stars. Young stars in comet-shaped clouds are therefore likely to have been formed by a triggering mechanism.

Alcalá et al. (2004) reported a sample of 33 young stellar objects (YSOs) associated with L1615/L1616, while Gandolfi et al. (2008) performed a comprehensive census of the pre-mainsequence (PMS) population in L1615/L1616, which consists of 56 YSOs. These two works were focused on the investigation

* Based on FLAMES (UVES+GIRAFFE) observations collected at the Very Large Telescope (VLT; Paranal, Chile). Program 076.C0385(A).

$\star \star$ Tables 3-6 and Appendices are available in electronic form at http://www . aanda.org of the star formation history, the relevance of the triggered scenario, and the initial mass function, but no study on accretion properties was addressed. As a continuation of these works, here we use further spectroscopic observations to derive the accretion luminosity, $L_{\text {acc }}$, and the mass accretion rate, $\dot{M}_{\text {acc }}$, of a sample of low-mass YSOs in L1615/L1616. We also investigate whether the accretion properties of YSOs in a cometary cloud like L1615/L1616 are similar to those of PMS stars in T associations, like Lupus, Taurus or Chamaeleon.

The outline of the paper is as follows. In Sect. 2, we describe the spectroscopic observations, the data reduction, and the sample investigated. In Sect. 3, several accretion diagnostics are used to derive the mass accretion rates. The main results on the accretion and infrared (IR) properties are discussed in Sect. 4, while our conclusions are presented in Sect. $5^{1}$.

\section{Observations, data reduction, and the sample}

\subsection{FLAMES observations and data reduction}

We conducted the observations in February-March 2006 in visitor mode using FLAMES (UVES+GIRAFFE) at the VLT. We used the CD\#3 cross-disperser and the LR6 grating for the UVES and GIRAFFE spectrographs, respectively. A brief summary of the observations is reported in Table 1, while the

\footnotetext{
Three appendices present additional material on: radial velocity and lithium abundance measurements, comparison between $\dot{M}_{\text {acc }}$ derived through three different PMS evolutionary tracks, and notes on individual objects.
} 
Table 1. Summary of the observations.

\begin{tabular}{lcccc}
\hline \hline Instrument & $\begin{array}{c}\text { Range } \\
(\AA)\end{array}$ & $\begin{array}{c}\text { Resolution } \\
(\lambda / \Delta \lambda)\end{array}$ & \# Stars & \# Spectra \\
\hline UVES & $4764-6820$ & 47000 & 1 & 6 \\
GIRAFFE & $6438-7184$ & 8600 & 23 & 53 \\
\hline
\end{tabular}

complete journal of the observations is listed in Table 3 . We observed 23 low-mass $\left(0.1 \lesssim M_{\star} \lesssim 2.3 M_{\odot}\right)$ objects with GIRAFFE in the MEDUSA mode ${ }^{2}$; one target (the classical T Tauri star - CTTs - LkH $\alpha 333$ ) was observed with both spectrographs. Nineteen objects were observed several (two-seven) times within two days (see Table 3).

We performed the reduction of the UVES spectra using the pipeline developed by Modigliani et al. (2004), which includes the following steps: subtraction of a master bias, échelle order definition, extraction of thorium-argon spectra, normalization of a master flat-field, frame extraction, wavelength calibration, and correction of the science frame for the normalized master flatfield. We performed the sky subtraction with the $\operatorname{IRAF}^{3}$ task SARITH using the fibers allocated to the sky.

We reduced the GIRAFFE data using the GIRAFFE BaseLine Data Reduction Software 1.13.1 (girBLDRS; Blecha et al. 2000): we performed bias and flat-field subtraction, correction for the fiber transmission coefficient, wavelength calibration, and science frame extraction. Then, we applied a sky correction to each stellar spectrum using the task SARITH in the IRAF ECHELLE package and by subtracting the average of several sky spectra obtained simultaneously during a given night.

\subsection{The sample}

Since our goal is to investigate the accretion and the IR properties of the YSOs in the cometary cloud, we need a wellcharacterized sample of YSOs both in terms of their physical parameters and their association with the cloud, as well as in terms of their accretion diagnostics and IR colors.

The stellar parameters (spectral types, effective temperatures, luminosities, and masses) were derived by Gandolfi et al. (2008). We adopt those determinations here. We note that one object, namely TTS 050730.9-031846, has a significantly lower luminosity compared to the other objects in the sample (see Fig. 3 in Gandolfi et al. 2008). This most probable subluminous object is further discussed in Appendix C.

Regarding the association with the cloud, we investigated the kinematics by means of radial velocity (RV) determinations, $V_{\text {rad }}$, and lithium abundance, $\log n(\mathrm{Li})$, following the same methods as in Biazzo et al. (2012). The details of such determinations can be found in Appendix A.1. The radial velocity distribution of the YSOs in L1615/L1616 has an average of $\left\langle V_{\mathrm{rad}}\right\rangle=$ $23.2 \pm 3.1 \mathrm{~km} \mathrm{~s}^{-1}$ (see Fig. A.1), which is consistent with that reported by Alcalá et al. (2004) (i.e., $\left\langle V_{\text {rad }}\right\rangle=22.3 \pm 4.6 \mathrm{~km} \mathrm{~s}^{-1}$ ), and in general with that of the Orion complex (see, e.g., Briceño 2008; Biazzo et al. 2009; Sergison et al. 2013). Likewise, the

\footnotetext{
2 This is the observing mode of FLAMES in which 132 fibers each with a projected diameter on the sky of 1".2, feed the GIRAFFE spectrograph. Some fibers are set on the target stars and others on the sky background.

3 IRAF is distributed by the National Optical Astronomy Observatory, which is operated by the Association of the Universities for Research in Astronomy, Inc. (AURA) under cooperative agreement with the National Science Foundation.
}

average lithium abundance is $\log n(\mathrm{Li}) \sim 3.3$ dex with a dispersion of \pm 0.3 dex (see Table 3 and Appendix A.2). Both radial velocities and lithium abundances confirm that all targets studied in this work are associated with the cometary cloud.

In order to have a more complete sample, we included in our analysis the YSOs lacking FLAMES spectroscopy, but for which Gandolfi et al. (2008) have provided measurements of the $\mathrm{H} \alpha$ equivalent width from FORS2 at VLT and VIMOS at VLT low-resolution spectra acquired in February-March 2003. Figure 1 shows the comparison between our $\mathrm{H} \alpha$ equivalent widths ${ }^{4}\left(\mathrm{EW}_{\mathrm{H} \alpha}\right)$ and the measurements reported by Gandolfi et al. (2008) for the stars in common. Although there is a general agreement, the Gandolfi et al. (2008) $\mathrm{EW}_{\mathrm{H} \alpha}$ are systematically higher than ours on average by about $4 \AA$ (excluding the three most deviating stars). We believe that this systematic difference is due to the lower spectral resolution used by Gandolfi et al. (2008) with respect to the resolution of our FLAMES spectra, whereas the differences are most likely related to variability for the three YSOs that deviate significantly from the 1:1 relationship in Fig. 1. Two of these objects will result to be accretors (see later on) and their variability will be discussed in Appendices C.1 and C.3, while the other is a weak lined T Tauri star (WTTs). The difference of $\sim 10 \AA$ between our $\mathrm{EW}_{\mathrm{H} \alpha}$ and the values by Gandolfi et al. (2008) could be related to stellar activity phenomena. This comparison justifies in the following analysis the use of the Gandolfi et al. (2008) $\mathrm{EW}_{\mathrm{H} \alpha}$ values for the stars we did not observed. Therefore, our analysis is based on 23 objects that we observed with FLAMES, and 31 targets previously observed by Gandolfi et al. (2008) at low resolution. All these objects are listed in Tables 4 and 5.

We used the criteria of White \& Basri (2003) based on spectral types and $\mathrm{EW}_{\mathrm{H} \alpha}$ to distinguish between accretors and nonaccretors. In this way, a total of 15 YSOs in L1615/L1616 can be classified as accretors, seven within our sample and eight within the Gandolfi et al. (2008) sample. Note that TTS 050649.8-031933, originally classified as a WTTs by Gandolfi et al. (2008) is tagged here as accretor because its spectrum shows helium and forbidden oxygen lines in emission (see Appendix C.2), typical of accreting objects (see their Table 4).

In addition, we considered ancillary IR data both from the Two-Micron All Sky Survey (2MASS; Cutri et al. 2003) and from the Wide-field Infrared Survey Explorer (WISE; Cutri et al. 2012) catalogs (see Table 4) to investigate the IR properties of the sample and their possible correlation with accretion diagnostics.

The position in the 2MASS color-color diagram of all YSOs classified here as accretors and nonaccretors is shown in Fig. 2 (filled and opened symbols, respectively). All YSOs we classified as accretors show near-IR excess. The anomalous colors of TTS 050730.9-031846 are consistent with its subluminous nature (see Sect. C.1). The three nonaccretors with apparent infrared excess represent stars with high values of $A_{V}$, as reported by Gandolfi et al. (2008). Their WISE colors are typical of Class III objects, confirming their WTTs nature (see Fig. 3). Moreover, note that all YSOs classified here as accretors have WISE colors typical of Class II YSOs.

Summarizing, we find a fraction of accretors in L1615/L1616 ( 30\%) consistent within the errors with the

\footnotetext{
4 We measured the equivalent widths of all lines used in the present work as accretor diagnostics with direct integration through the IRAF task SPLOT. We adopted the standard deviations of three measurements as errors in the line equivalent widths.
} 
K. Biazzo et al.: On the accretion properties of young stellar objects in the L1615/L1616 cometary cloud

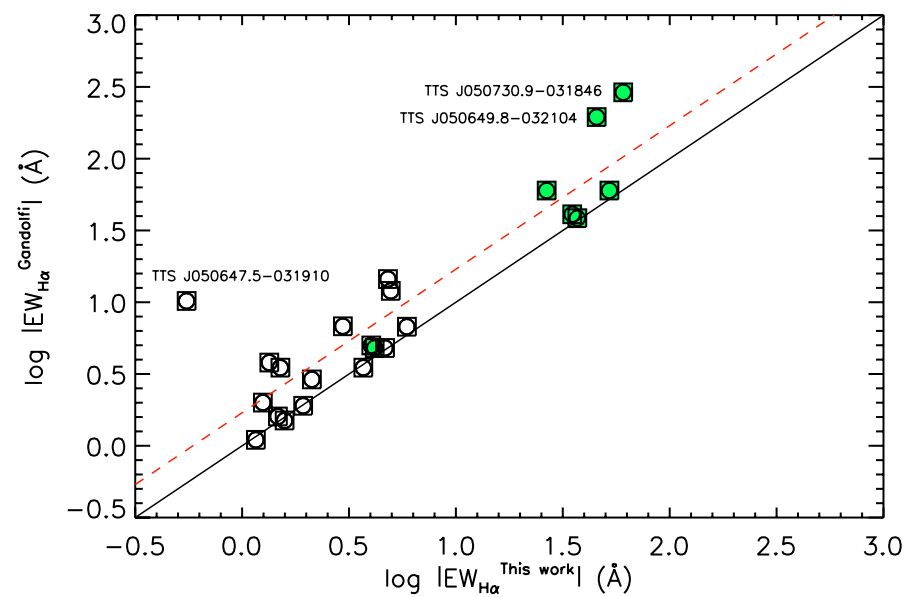

Fig. 1. Comparison between our $\mathrm{EW}_{\mathrm{H} \alpha}$ values and those obtained by Gandolfi et al. (2008). The solid line represents the 1:1 relation, while the dashed line is shifted by the rms difference between the $\log E W_{\mathrm{H} \alpha}$ values (excluding the three most deviating objects labeled in the figure). Filled symbols refer to most probable accreting stars (see text and Fig. 2).

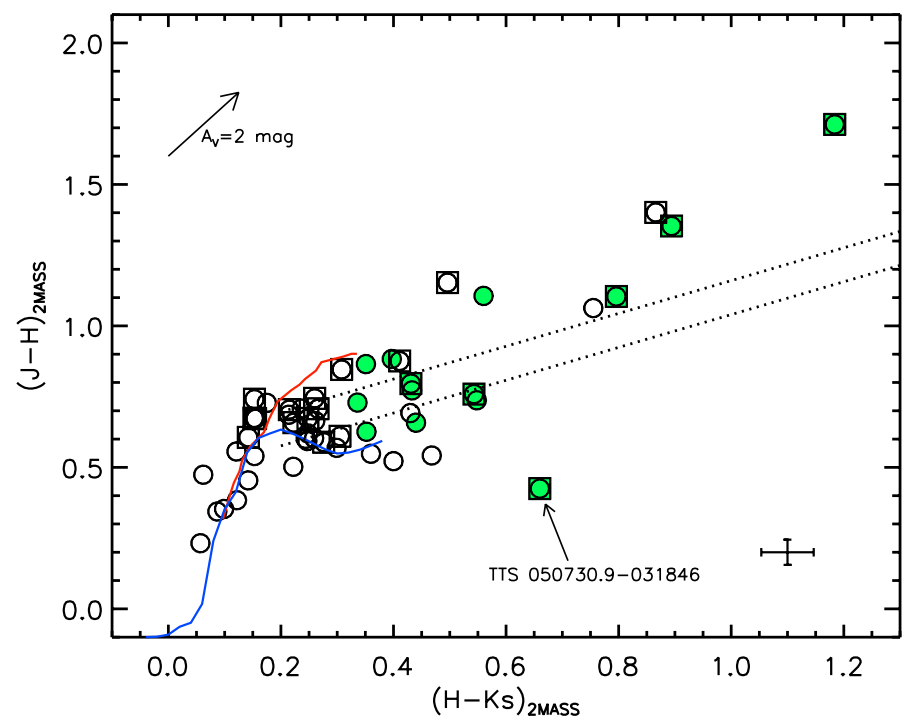

Fig. 2. 2MASS color-color diagram of the L1615/L1616 targets. Open circles represent low-mass stars analyzed by Gandolfi et al. (2008), while squares mark the targets observed for this work with the FLAMES spectrograph. Filled symbols are the most probable accretors as defined in Sect. 2.2. The dwarf (lower branch; Bessell \& Brett 1988) and the giant (upper branch; Kenyon \& Hartmann 1995) sequences are marked by solid lines. The arrow at the top upper-left corner indicates the reddening vector for $A_{V}=2 \mathrm{mag}$. The CTTs locus (Meyer et al. 1997) is delimited by the dotted lines. The position of the subluminous candidate TTS $050730.9-031846$ is also displayed. The mean 2MASS photometric errors are overplotted on the lower-right corner of the panel.

fraction of disks recently reported by Ribas et al. (2014) for an average age of $3 \mathrm{Myr}$ (see their Fig. 2).

\section{Accretion diagnostics and mass accretion rates}

According to the magnetospheric accretion model (Uchida \& Shibata 1985; Königl 1991; Shu et al. 1994), matter is accreted from the disk onto the star and shocks the stellar surface producing high temperature $\left(\sim 10^{4} \mathrm{~K}\right)$ gas, giving rise to emission in the blue continuum and in many lines, which can be observed as photometric and spectroscopic diagnostics. Primary accretion diagnostics, such as the UV excess emission, the Paschen/Balmer continua, and the Balmer jump (see, e.g., Herczeg \& Hillenbrand 2008; Alcalá et al. 2014), and secondary tracers, like hydrogen recombination lines and the He I, Ca II, $\mathrm{Na}$ I lines (see, e.g., Muzerolle et al. 1998; Antoniucci et al. 2011; Biazzo et al. 2012) are therefore useful tools to detect accretion signatures and to derive the energy losses due to accretion, i.e., the accretion luminosity (e.g., Gullbring et al. 1998; Herczeg \& Hillenbrand 2008; Rigliaco et al. 2011b; Ingleby et al. 2013; Alcalá et al. 2014).

In the context of the magnetospheric accreting model, the accretion luminosity can be converted into mass accretion rate, $\dot{M}_{\text {acc }}$, using the following relationship (Hartmann 1998):

$\dot{M}_{\mathrm{acc}}=\left(1-\frac{R_{\star}}{R_{\mathrm{in}}}\right)^{-1} \frac{L_{\mathrm{acc}} R_{\star}}{G M_{\star}} \approx 1.25 \frac{L_{\mathrm{acc}} R_{\star}}{G M_{\star}}$,

where $M_{\star}$ and $R_{\star}$ are the stellar mass and radius, respectively, $R_{\text {in }}$ is the YSO inner disk radius, and $G$ is the universal gravitational constant. The inner radius corresponds to the distance at which the disk is truncated, due to the stellar magnetosphere, and from which the disk gas is accreted, and channeled by the magnetic field lines. In previous works, it has been assumed that $R_{\text {in }}$ is $\sim 5 R_{\star}$ (see, e.g., Alcalá et al. 2011).

The accretion luminosity can be estimated from empirical linear relationships between the observed line luminosity, $L^{\lambda}$, and $L_{\text {acc }}$ derived through primary diagnostics (see, e.g., Gullbring et al. 1998; Herczeg \& Hillenbrand 2008; Alcalá et al. 2014). Such relationships have been established by the simultaneous observations of many accretion indicators and by modeling the continuum excess emission.

For the accretors in our sample, we used the luminosity of several emission lines $(\mathrm{H} \alpha \lambda 6563 \AA, \mathrm{H} \beta \lambda 4861 \AA$, He I $\lambda 5876 \AA$, He I $\lambda 6678 \AA$, and He I $\lambda 7065 \AA$ ) within the wavelength range covered by the FLAMES spectra, while for the objects in Gandolfi et al. (2008) we used the $\mathrm{H} \alpha$ line. We then considered the recent $L^{\lambda}-L_{\text {acc }}$ relations by Alcalá et al. (2014) to derive the accretion luminosity from each line. These relationships consider a combination of all accretion indicators calibrated on sources for which the UV excess emission and the Paschen/Balmer continua were measured simultaneously.

Unfortunately, we do not have simultaneous or quasisimultaneous photometry in hand and our fiber-fed spectra cannot be calibrated in flux. Therefore, the best approach to calculate line luminosities from our data is by deriving line surface fluxes using equivalent widths and assuming continuum fluxes from model atmospheres. Thus, we calculated the line luminosity $L^{\lambda}$ using the same approach as in Biazzo et al. (2012). In particular, $L^{\lambda}=4 \pi R_{\star}^{2} F^{\lambda}$, where we took the stellar radius from Gandolfi et al. (2008) and we derived the surface flux, $F^{\lambda}$, by multiplying the $\mathrm{EW}$ of each line $\left(\mathrm{EW}_{\lambda}\right)$ by the continuum flux at wavelengths adjacent to the line $\left(F_{\text {cont }}^{\lambda \pm \lambda}\right)$. We gathered the latter from the NextGen Model Atmospheres (Hauschildt et al. 1999), assuming the corresponding YSO effective temperature and surface gravity. We estimated the gravity for every YSO from the mass and stellar radius reported in Gandolfi et al. (2008). In particular, we considered the three different $M_{\star}$ values provided by the authors for three different sets of PMS evolutionary tracks (namely, Baraffe et al. 1998 and Chabrier et al. 2000; D’Antona \& Mazzitelli 1997; Palla \& Stahler 1999; hereafter Ba98+Ch00, DM97, PS99, respectively, as used in Gandolfi et al. 2008). We stress that the mean difference in $\log g$ coming from the use of the three evolutionary tracks varies from $\sim 0.0$ to 0.4 dex. Such 
differences in $\log g$ may lead to an uncertainty in the continuum flux of less than $\sim 10 \%$, mainly depending on the effective temperature, the surface gravity itself, and the line considered. This represents the typical error in the continuum flux we considered for the estimation of the uncertainty in $\dot{M}_{\text {acc }}$ (see text later on).

In the end, the different line diagnostics we considered yielded consistent values of $L_{\text {acc }}$, which justified the use of all of them to compute an average $\left\langle L_{\text {acc }}\right\rangle$ for each YSO (see Table 6). In this way, the error on the average $L_{\text {acc }}$ derived from several diagnostics, measured simultaneously, is minimized, as found by Rigliaco et al. (2012) and Alcalá et al. (2014). We then calculated the mass accretion rate $\left(\dot{M}_{\text {acc }}\right)$ using $\left\langle L_{\text {acc }}\right\rangle$ and the Eq. (1), and adopting the $M_{\star}$ and $R_{\star}$ values reported in Gandolfi et al. (2008). For every accretor, we thus derived three values of $\dot{M}_{\text {acc }}$ using the three values of $M_{\star}$ (Table 6).

Contributions to the error budget on $\dot{M}_{\text {acc }}$ include uncertainties in stellar mass, stellar radius, inner disk radius, and $L_{\text {acc }}$. Assuming mean errors of $\sim 0.1 M_{\odot}$ in $M_{\star}$ and $\sim 0.1 R_{\odot}$ in $R_{\star}$ (Gandolfi et al. 2008), 1-13\% as relative error in $\mathrm{EW}_{\lambda}, 10 \%$ in $F_{\text {cont }}^{\lambda \pm \Delta \lambda}$, and the uncertainties in the relationships by Alcalá et al. (2014), we estimate a typical error in $\log \dot{M}_{\text {acc }}$ of $\sim 0.5 \mathrm{dex}$.

Note that the equivalent width values are not corrected for veiling, which alters the continuum of the spectra. In case of strong accretors, the continuum excess emission becomes important, but we quantify this effect on our sample in the next section.

\subsection{Impact of veiling on the $\dot{M}_{a c c}$ estimates}

We estimate how the amount of veiling affects the $\dot{M}_{\text {acc }}$ estimates by running the ROTFIT ${ }^{5}$ code (Frasca et al. 2003, 2006) on the spectra of our accretors. This code compares the target spectrum with a grid of slowly rotating and low activity templates, aligned with the target spectrum, re-sampled on its spectral points, and artificially broadened with a rotational profile until the minimum of the residuals is reached (see details in Frasca et al. 2014). In order to find the best template to reproduce the veiled accretors, we included the veiling $(r)$ as an additional parameter. We made this by adding a featureless veiling to each template, whose continuum-normalized spectrum becomes

$$
\left(\frac{F_{\lambda}}{F_{\text {cont }}}\right)^{r}=\frac{\left(\frac{F_{\lambda}}{F_{\text {cont }}}\right)+r}{1+r} .
$$

This procedure could be applied only to five accretors in our sample. Unfortunately, the low resolution of the spectra acquired by Gandolfi et al. (2008) and the very low signal-to-noise ratio $(\mathrm{S} / \mathrm{N})$ of some FLAMES spectra were not sufficient to apply our method.

In Fig. 4, we show an example of an accreting star observed with UVES ( $\mathrm{LkH} \alpha 333)$, with $r=0.5$, as found by ROTFIT. In Table 2, we list the mean veiling derived from the FLAMES spectra. Using these values, we could estimate the new $\dot{M}_{\text {acc }}$ correcting the measured EWs of the lines by the factor $(1+r)$. We can conclude that the correction for the veiling leads to a difference of $\sim 0.25 \mathrm{dex}$ in $\log \dot{M}_{\text {acc }}$ at most (i.e., they are within the errors of our estimates and do not affect our conclusions). Similar results were also found by Costigan et al. (2012). Hereafter, as we could not evaluate the veiling for all our targets, we will adopt the $\dot{M}_{\text {acc }}$ without any correction for the veiling.

\footnotetext{
5 ROTFIT is an IDL code. IDL (Interactive Data Language) is a registered trademark of Exelis Visual Information Solutions.
}

Table 2. Mean veiling and difference in $\log \dot{M}_{\text {acc }}$ for the targets for which we ran the ROTFIT code.

\begin{tabular}{lcc}
\hline \hline ID Name & $r$ & $\begin{array}{c}\Delta \log \dot{M}_{\text {acc }} \\
(\mathrm{dex})\end{array}$ \\
\hline TTS J050646.1-031922 & 0.50 & 0.25 \\
RX J0506.9-0319 SE & 0.25 & 0.10 \\
LkH $\alpha$ 333 & 0.50 & 0.20 \\
L1616 MIR 4 & 0.50 & 0.20 \\
RX J0507.1-0321 & 0.50 & 0.20 \\
\hline
\end{tabular}

\subsection{Variability}

Being based on single "epoch" measurements of line equivalent widths and estimates of continuum fluxes, our calculations of line luminosity and accretion luminosity represent only an instantaneous snapshot of $L_{\text {acc }}$ and $\dot{M}_{\text {acc }}$. As in previous investigations in other star-forming regions (SFRs; see, e.g., Nguyen et al. 2009; Biazzo et al. 2012; Costigan et al. 2012, 2014; Fang et al. 2013), and based on multiepoch observations of several of our targets, we estimate that short timescale $(\sim 48 \mathrm{~h})$ variations may induce a scatter on $\log \dot{M}_{\text {acc }}$ of $<0.3 \mathrm{dex}$, while at a longer timescale (a few years) variations may be up to $\sim 0.6 \mathrm{dex}$ (see Appendix C.3). Therefore, as claimed in those studies, we also conclude that YSOs variability may account for variations in $\log \dot{M}_{\text {acc }}$ in the range of $\sim 0.2-0.6$ dex.

\section{Results and discussion}

In the following, we discuss the accretion properties of the sample and their link to the stellar parameters and the IR colors.

\subsection{Accretion luminosity versus stellar parameters}

At low levels of accretion, the chromospheric emission may have an important impact on the estimates of $L_{\text {acc }}$ (see Manara et al. 2013, and references therein). This contribution should be therefore considered when accretion properties are studied. As shown in Fig. 5, the accretion luminosity of the YSOs in L1615/L1516 decreases monotonically with the effective temperature. The dashed line in this figure shows the chromospheric level as determined by Manara et al. (2013), and represents the locus below which the contribution of chromospheric emission starts to be important in comparison with energy losses due to accretion. All accreting YSOs in L1615/L1616 fall well above the "systematic noise" due to chromospheric emission and show $L_{\text {acc }} / L_{\odot}$ very similar to the values recently derived for members of the Lupus SFR by Alcalá et al. (2014) and estimated through primary diagnostics.

Figure 6 shows the mean accretion luminosity as a function of the stellar luminosity. As already observed by previous investigations in other SFRs, such as $\rho$ Ophiucus, Taurus, and Lupus (Muzerolle et al. 1998; Natta et al. 2006; Alcalá et al. 2014), the accretion luminosity increases with the stellar luminosity. In our sample of accreting stars, $L_{\text {acc }}$ follows a trend that is similar to the recent power law found by Alcalá et al. (2014) in the Lupus star-forming region. Moreover, the dispersion of our data points in $L_{\text {acc }}$ is similar. As in other star-forming regions, the accretion luminosity of the YSOs in L1615/L1616 is a fraction of the stellar luminosity, and falls in the range between $0.1 L_{\star}$ to $0.01 L_{\star}$ (see, e.g., Muzerolle et al. 1998; White \& Hillenbrand 2004; Antoniucci et al. 2011; Caratti o Garatti et al. 2012; Alcalá et al. 2014). 
K. Biazzo et al.: On the accretion properties of young stellar objects in the L1615/L1616 cometary cloud
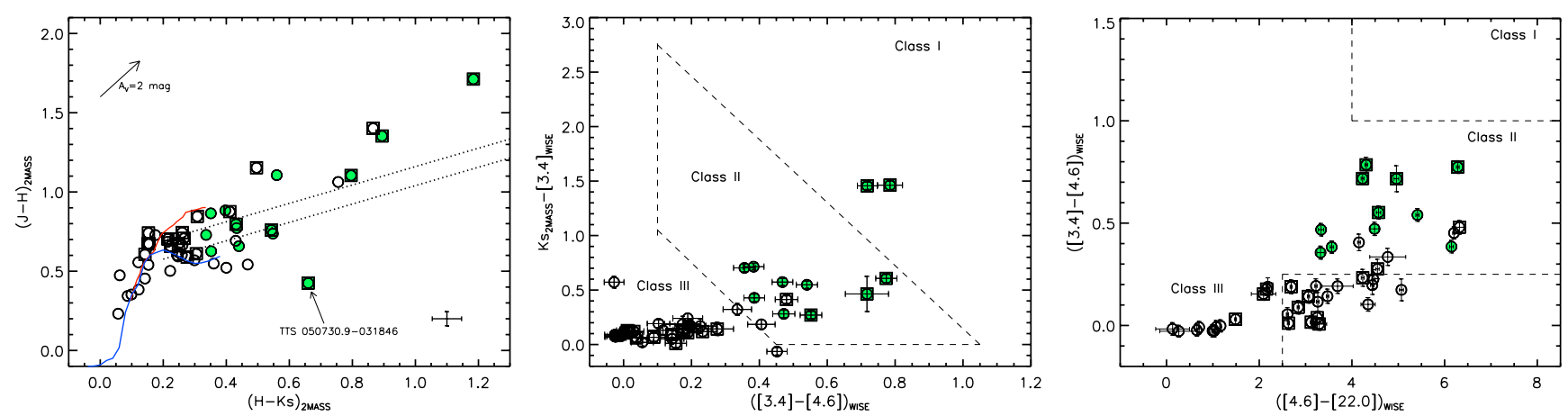

Fig. 3. WISE/2MASS color-color diagrams of the L1615/L1616 targets. Dashed lines indicate the boundaries of Class I, Class II, and diskless stars (Class III objects) as defined in Koenig et al. (2012). The nonaccretors falling within the Class II region will be discussed in Appendix C. Symbols are as in Fig. 2.
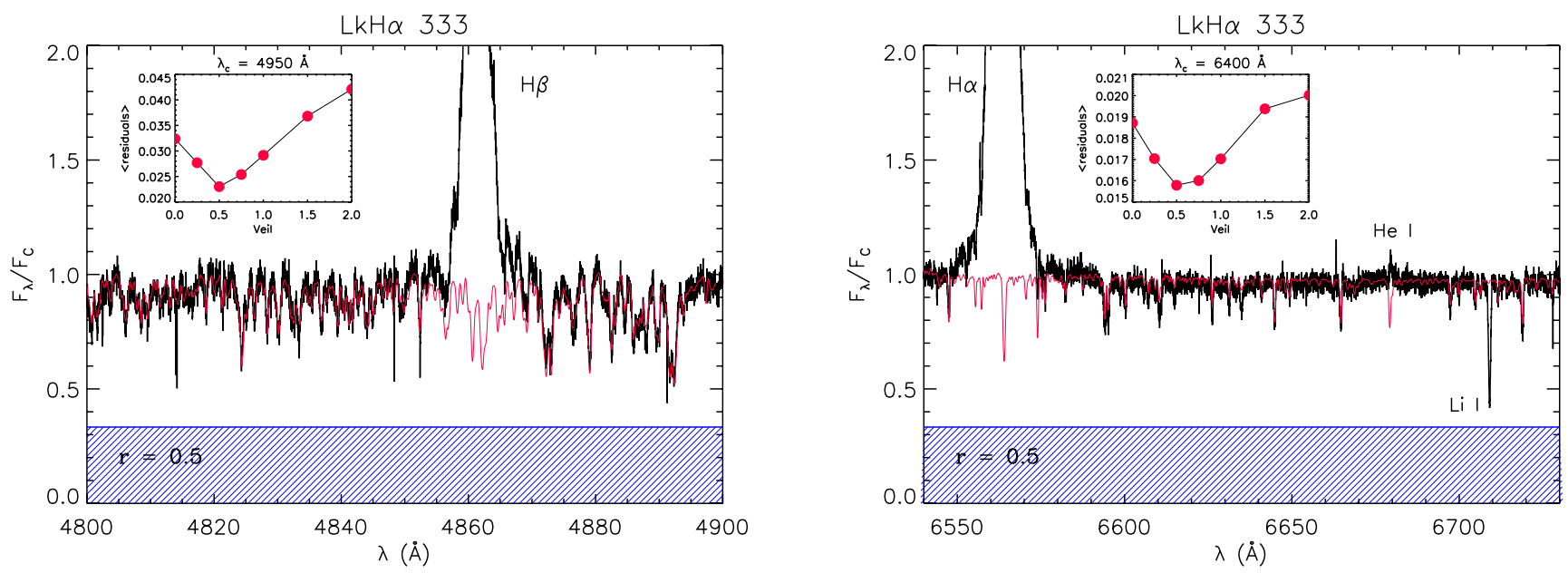

Fig. 4. Example of UVES spectrum of the accreting star $\mathrm{LkH} \alpha 333$ both in the $\mathrm{H} \beta$ (left panel) and in the $\mathrm{H} \alpha$ (right panel) region. In both panels, the black solid line represents the spectrum of the target, while the red solid line is the spectrum of the best template reproducing the target at $r=0.5$. The residuals as a function of the $r$ parameter are plotted in the inset. The hatched area is the level of the veiling at 0.5 .

\subsection{Mass accretion rate versus stellar mass}

The distribution of YSOs in the $\dot{M}_{\text {acc }}$ versus $M_{\star}$ plane provides an important diagnostic for the studies of the evolution of mass accretion (see Hartmann et al. 2006). The $\dot{M}_{\text {acc }}$ versus $M_{\star}$ relationship has been obtained for a number of different star-forming regions (e.g., Taurus, Ophiuchus, $\sigma$ Orionis, Orion Nebula Cluster, Trumpler 37). In all regions studied so far, it has been found that although there is a rough correlation of $\dot{M}_{\text {acc }}$ with the square of $M_{\star}$, the scatter of $\dot{M}_{\text {acc }}$ for a given mass is very large (e.g., Muzerolle et al. 2005; Natta et al. 2006).

The physical origin of the $\dot{M}_{\text {acc }} \propto M_{\star}^{\alpha}$ relationship, with $\alpha \approx 2$, is still unclear. Alexander \& Armitage (2006) have suggested that the correlation reflects the initial conditions established when the disk formed, followed by subsequent viscous disk evolution. The natural decline of the mass accretion rate with age in viscous disk evolution and effects due to evolutionary differences within a sample have been ruled out as possible cause for the large spread of the relationship within individual star-forming regions (Mohanty et al. 2005; Natta et al. 2006). Moreover, short-term (see, e.g., Nguyen et al. 2009; Biazzo et al. 2012) and long-term variability may contribute to, but cannot explain the large vertical spread of the $\dot{M}_{\text {acc }}-M_{\star}$ relationship (Biazzo et al. 2012; Costigan et al. 2012, 2014). It appears more likely related to a spread in the properties of the parental cores, their angular momentum in particular (e.g.,
Hartmann et al. 2006; Dullemond et al. 2006), in stellar properties, such as X-ray emission (Muzerolle et al. 2003), or to the competition between different accretion mechanisms, such as viscosity and gravitational instabilities (Vorobyov \& Basu 2008). As opposed to Dullemond et al. (2006), Ercolano et al. (2014) argued that the $\dot{M}_{\text {acc }}$ versus $M_{\star}$ relation for a population of disks dispersing via X-ray photoevaporation is completely determined by the shape of the X-ray luminosity function, hence requires no spread in initial conditions other than the dependence on stellar mass. On the other hand, Alcalá et al. (2014) have concluded that mixing mass accretion rates calculated with different techniques may increase the scatter in the $\dot{M}_{\text {acc }}$ versus $M_{\star}$ relationship. They also have claimed that different methodologies used to derive accretion luminosity and line luminosity, as well as different evolutionary models used to estimate masses may lead to significantly different results on the slope of the relationship.

The results in the $\dot{M}_{\text {acc }}-M_{\star}$ plane for our sample of accretors in L1615/L1616 are shown in Fig. 7. The three panels in this figure correspond to the values of $\dot{M}_{\text {acc }}$ calculated from the three estimates of $M_{\star}$ drawn from the three evolutionary models, as labeled in the figure. Since our statistics are poor, we do not attempt a linear fit to the $\dot{M}_{\text {acc }}-M_{\star}$ relationships. Instead, for comparison, we overplot the $\dot{M}_{\text {acc }}-M_{\star}$ linear fit with a slope of $1.8 \pm 0.2$ recently calculated for Lupus YSOs (Alcalá et al. 2014), for which the accretion luminosity was directly 


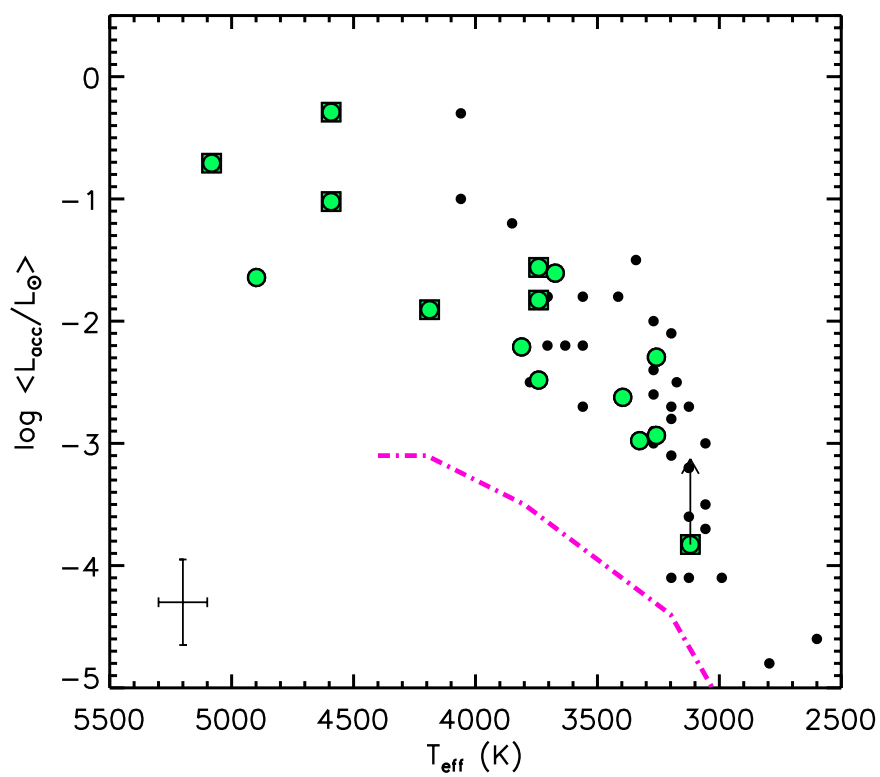

Fig. 5. Mean accretion luminosity versus effective temperature. Dashdotted line marks the locus below which chromospheric emission is important in comparison with $L_{\text {acc }}$ (Manara et al. 2013). The vertical arrow represents the position of the subluminous object after applying the correction factor described in Appendix C.1. Small filled dots represent the Alcalá et al. (2014) sample of low-mass stars in the Lupus SFR. Mean error bars are overplotted on the lower-left corner of the panel. Symbols are as in Fig. 2.

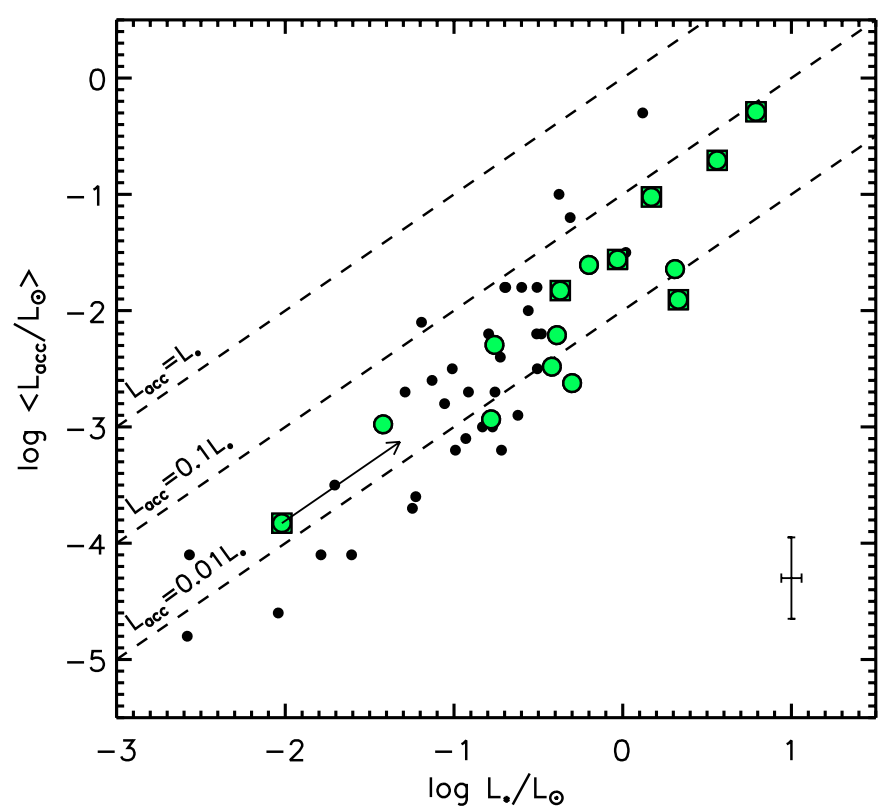

Fig. 6. Mean accretion luminosity versus stellar luminosity. Dashed lines represent the loci of the three $L_{\text {acc }}-L_{\star}$ relations, as labeled. The arrow represents the position of the subluminous object after applying the correction factor described in Appendix C.1. The Lupus YSOs by Alcalá et al. (2014) are overlaid as small filled dots. Mean error bars are overplotted on the lower-right corner of the panel. Symbols are as in Fig. 2.

derived by modeling the excess emission from the UV to the near-infrared as the continuum emission of from a slab of hydrogen. Similar findings were also obtained in other T associations, like Taurus or Chamaeleon (see, e.g., Herczeg \& Hillenbrand 2008; Antoniucci et al. 2011; Biazzo et al. 2012).
The accretors in L1615/L1616 closely follow the $\dot{M}_{\text {acc }}-M_{\star}$ relationship seen for the YSOs in Lupus by Alcalá et al. (2014), but interestingly the scatter changes depending on the evolutionary tracks used to derive the stellar masses. As concluded in Appendix B, the different evolutionary tracks have negligible effects on the computation of $\log \dot{M}_{\text {acc }}$, meaning that the scatter in the $\log \dot{M}_{\text {acc }}-\log M_{\star}$ diagram is mainly induced by the uncertainty in the mass, which is model-dependent. The DM97 tracks seem to produce the largest scatter.

We stress, however, that some scatter in the $\dot{M}_{\text {acc }}-M_{\star}$ relationship (up to around $\pm 0.5-0.6 \mathrm{dex}$ in $\log \dot{M}_{\text {acc }}$; see, e.g., Costigan et al. 2014, and references therein) may come from intrinsic variability, as our line luminosity determinations were obtained from single "epoch" measurements of line equivalent widths and assumed continuum flux coming from model atmospheres (see Sect. 3.2).

\subsection{Accretion versus infrared properties}

Near- and mid-IR colors can be used to probe the inner disk region. Hartigan et al. (1995), studying a sample of $42 \mathrm{~T}$ Tauri stars and using the $K-L$ color excesses, pointed out that disk dissipation is mainly due to the formation of micron-sized dust particles, which merge to create planetesimals and protoplanets at the end of the CTTs phase. Protoplanets may clear the innermost part of the disk where the gas and dust have temperatures of the order of $\sim 1000 \mathrm{~K}$ and emit mainly at near-IR and midIR wavelengths. This causes the disk to decrease or loose its near-IR color excess and, at the same time, the opening of a gap in the disk (see, e.g., Lin \& Papaloizou 1993), thereby possibly terminating accretion from the disk onto the star.

With the aim of investigating possible relationships between IR colors and accretion properties, we plotted in Fig. 8 the $J-H$, $H-K \mathrm{~s}, J-K \mathrm{~s} 2 \mathrm{MASS}$ colors and the [3.4]-[4.6], [4.6]-[12.0], [4.6]-[22.0] WISE colors as a function of the mass accretion rates derived using the PS99 tracks. From these tracks, masses could be estimated for all the accretors we studied in this work. Despite poor statistics, the behavior of the 2MASS and WISE colors with accretion is different. While the 2MASS colors tend to rise at $\dot{M}_{\text {acc }} \gtrsim 10^{-10} M_{\odot} \mathrm{yr}^{-1}$, the WISE colors show no trend with $\dot{M}_{\text {acc }}$. In order to quantify the degree of possible correlations in Fig. 8, we computed the Spearman's rank correlation coefficients with the IDL platform. These correlation coefficients, ranging from 0 to 1 , show values around 0.6 for the relations between $\dot{M}_{\text {acc }}$ and 2 MASS colors, and values very close to 0.0 for the WISE colors, meaning that the 2MASS colors show an increasing trend with $\log \dot{M}_{\text {acc }}$, while no trend is detected for relations between $\dot{M}_{\text {acc }}$ and the WISE colors.

The trend between 2MASS colors and $\log \dot{M}_{\text {acc }}$ could be an indication that objects with strong accretion have optically thick inner disks, as found in previous works (Hartigan et al. 1995; Rigliaco et al. 2011a; Biazzo et al. 2012). In particular, we can identify the regions $\log \dot{M}_{\text {acc }} \gtrsim-8.5$ dex and $J-H \gtrsim 1.0$ or $H-K \mathrm{~s} \gtrsim 0.7$ or $J-K \mathrm{~s} \gtrsim 1.75$ as those where strong accreting objects with large near-IR excess are found in L1615/L1616.

\section{Conclusions}

In this paper, we investigated the accretion and the IR properties of YSOs in the cometary cloud L1615/L1616 in Orion. For this purpose, we used intermediate resolution (FLAMES at VLT) and low resolution (FORS2 at VLT + VIMOS at VLT) optical 
K. Biazzo et al.: On the accretion properties of young stellar objects in the L1615/L1616 cometary cloud
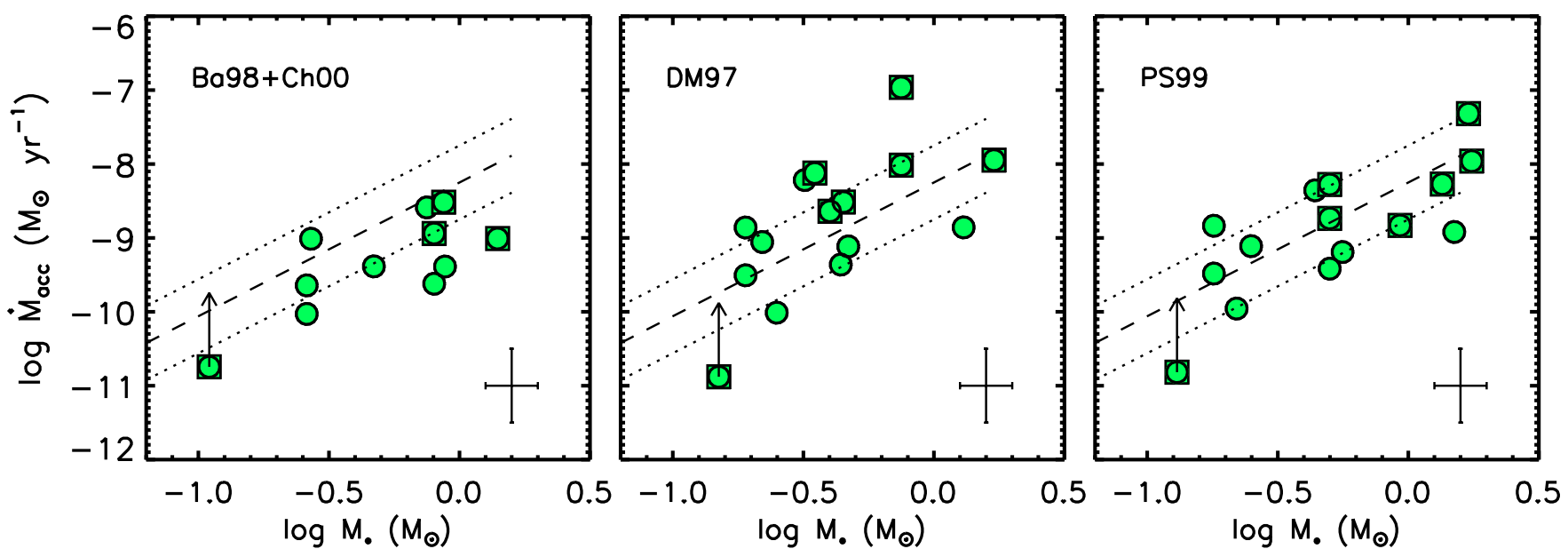

Fig. 7. Mass accretion rate versus stellar mass drawn from the Ba98+Ch00, DM97, and PS99 evolutionary tracks, respectively (from the left to the right panel). Dashed and dotted lines represent the $\dot{M}_{\text {acc }}-M_{\star}$ relationship and the $1 \sigma$ deviation from fit obtained with X-Shooter at VLT observations by Alcalá et al. (2014) for YSOs in Lupus. The vertical arrow represents the position of the subluminous object after applying the correction factor described in Appendix C.1. Mean error bars are overplotted on lower-right corner of each panel. Symbols are as in Fig. 2.
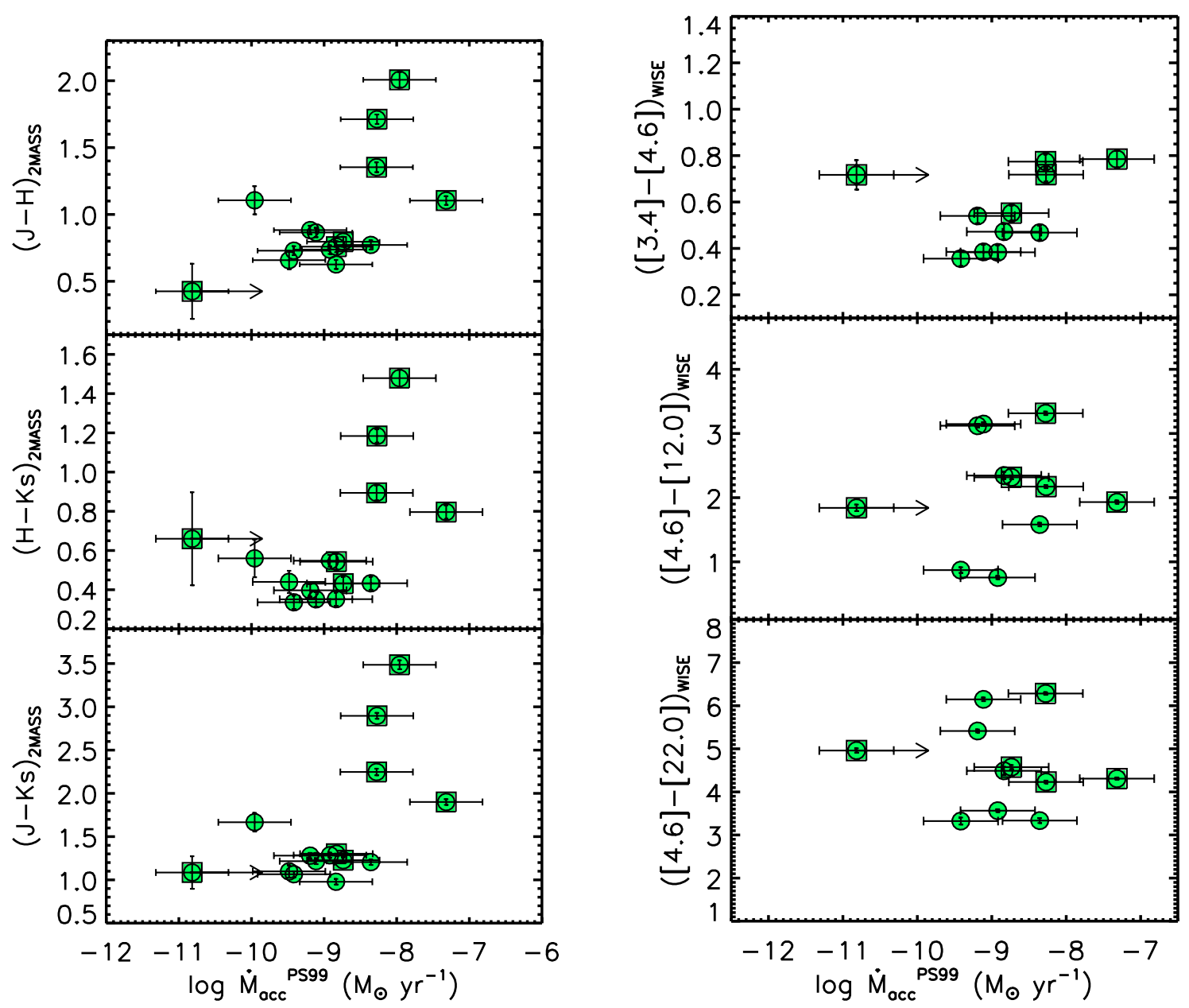

Fig. 8. 2MASS (left panel) and WISE (right panel) colors versus mass accretion rates derived using the PS99 stellar masses. The horizontal arrows represent the position of the subluminous object after correction of $\dot{M}_{\text {acc }}$, as explained in Appendix C.1. Symbols are as in Fig. 2.

spectroscopy for 23 and 31 objects, respectively. Our main results can be summarized as follows:

1. The YSOs in L1615/L1616 observed with FLAMES show a narrow distribution in radial velocity peaked at $23.2 \pm$ $3.1 \mathrm{~km} \mathrm{~s}^{-1}$, showing they are dynamically associated with the cloud. The YSOs also show the mean lithium abundance of $3.3 \pm 0.3$ dex confirming their membership to the cometary cloud and their youth.

2. The fraction of accretors in L1615/L1516 is close to $30 \%$, consistent with the fraction of disks recently reported by Ribas et al. (2014) for an average age of $3 \mathrm{Myr}$. 
3. The mass accretion rates $\left(\dot{M}_{\text {acc }}\right)$ derived through several secondary diagnostics $(\mathrm{H} \alpha, \mathrm{H} \beta, \mathrm{He} \mathrm{I} \lambda 5876 \AA$, He I $\lambda 6678 \AA$, and He I $\lambda 7065 \AA$ ) are in the range $\sim 10^{-10}-10^{-7} M_{\odot} \mathrm{yr}^{-1}$ for stars with $\sim 0.1-2.3 M_{\odot}$. These accretion rates are similar to those of YSOs of similar mass in other star-forming regions.

4. The accretion properties of the YSOs in L1615/L1616 exhibit the same behavior as YSOs in other star-forming regions, like Lupus or Taurus. This might imply that environmental conditions to which the cometary cloud is exposed do not influence the accretion evolution of the YSOs in this cometary cloud.

5. As recently found by other authors, we confirm that different methods used to derive stellar parameters and mass accretion rates introduce dispersion in the $\dot{M}_{\text {acc }}-M_{\star}$ relation; in particular, the differences in the evolutionary tracks used to derive $M_{\star}$ and then $\dot{M}_{\text {acc }}$ produce a scatter in the $\dot{M}_{\text {acc }}-M_{\star}$ relationship, but no significant systematic effect on $\dot{M}_{\text {acc }}$.

6. The color $-\dot{M}_{\text {acc }}$ diagrams suggest that strong accretors (i.e., those with $\log \dot{M}_{\text {acc }} \gtrsim-8.5$ dex) show large excesses in the $J H K$ s bands, indicative of inner optically thick disk, as in previous studies.

Acknowledgements. The authors are very grateful to the referee for carefully reading the paper and for his/her useful remarks that allowed us to improve the previous version of the manuscript. K.B. acknowledges the Osservatorio Astronomico di Capodimonte for the support given during her visits. This research made use of the SIMBAD database, operated at the CDS (Strasbourg, France). This publication makes use of data products from the Two Micron All Sky Survey, which is a joint project of the University of Massachusetts and the Infrared Processing and Analysis Center/California Institute of Technology, funded by NASA and the National Science Foundation. This publication makes use of data products from the Wide-field Infrared Survey Explorer, which is a joint project of the University of California, Los Angeles, and the Jet Propulsion Laboratory/California Institute of Technology, funded by the National Aeronautics and Space Administration. This work was financially supported by the PRIN INAF 2013 "Disks, jets and the dawn of planets".

\section{References}

Alcalá, J. M., Wachter, S., Covino, E., et al. 2004, A\&A, 416, 677

Alcalá, J. M., Covino, E., \& Leccia, S. 2008, in Handbook of Star Forming Regions, Vol. I: The Northern Sky ASP Monograph Publications, ed. B. Reipurth, 4, 801

Alcalá, J. M., Stelzer, B., Covino, E., et al. 2011, Astron. Nachr., 332, 242

Alcalá, J. M., Natta, A., Manara, C., et al. 2014, A\&A, 561, A2

Alexander, R. D., \& Armitage P. J. 2006, ApJ, 639, L83

Antoniucci, S., García-López, R., Nisini, B., et al. 2011, A\&A, 534, A32

Baraffe, I., \& Chabrier, G. 2010, A\&A, 521, A44

Baraffe, I., Chabrier, G., Allard, F., \& Hauschildt, P. H. 1998, A\&A, 337, 403

Bessell, M. S., \& Brett, J. M. 1988, PASP, 100, 1134

Biazzo, K., Melo, C. H. F., Pasquini, L., et al. 2009, A\&A, 508, 1301

Biazzo, K., Alcalá, J. M., Covino, E., et al. 2012, A\&A, 547, A104

Blecha, A., Cayatte, V., North, P., Royer, F., \& Simond, G. 2000, Proc. SPIE, 4008,467

Briceño, C. 2008, in Handbook of Star Forming Regions, Vol. I: The Northern Sky ASP Monograph Publications, ed. B. Reipurth, 4, 838

Caratti o Garatti, A., García-López, R., Antoniucci, S., et al. 2012, A\&A, 538, A64

Chabrier, G., Baraffe, I., Allard, F., \& Hauschildt, P. H. 2000, ApJ, 542, 464
Comerón, F., Fernández, M., Baraffe, I., Neuhäuser, R., \& Kaas, A. A. 2003, A\&A, 406, 1001

Costigan, G., Schölz, A., Stelzer, B., et al. 2012, MNRAS, 427, 1344

Costigan, G., Vink, Jorick S., Schölz, A., Ray, T., \& Testi, L. 2014, MNRAS, 440,3444

Cutri, R. M., Skrutskie, M. F., van Dyk, S., et al. 2003, Explanatory Supplement to the 2MASS All Sky Data Release

Cutri, R. M., Wright, E. L., Conrow, T., et al. 2012, Explanatory Supplement to the WISE All-Sky Data Release Products

D’Antona, F., \& Mazzitelli, I. 1997, Mem. Soc. Astron. It., 68, 807

Dullemond, C. P., Natta, A., \& Testi, L. 2006, ApJ, 645, 69

Ercolano, B., Mayr, D., Owen, J. E., Rosotti, G., \& Manara, C. F. 2014, MNRAS 439, 256

Fang, M., van Boekel, R., Wang, W., et al. 2009, A\&A, 504, 461

Fang, M., Kim, J. S., van Boekel, R., et al. 2013, ApJS, 207, 5

Frasca, A., Alcalá, J. M., Covino, E., et al. 2003, A\&A, 405, 149

Frasca, A., Guillout, P., Marilli, E., et al. 2006, A\&A, 454, 301

Frasca, A., Biazzo, K., Lanzafame, A., et al. 2014, A\&A, submitted

Gandolfi, D., Alcalá, J. M., Leccia, S., et al. 2008, ApJ, 687, 1303

Gullbring, E., Hartmann, L., Briceño, C., \& Calvet, N. 1998, ApJ, 492, 323

Hartigan, P., Edwards, S., \& Ghandour, L. 1995, ApJ, 452, 736

Hartmann, L. 1998, in Accretion Processes in Star Formation (Cambridge Univ. Press)

Hartmann, L., D’Alessio, P., Calvet, N., \& Muzerolle, J. 2006, ApJ, 648, 484

Hauschildt, P. H., Allard, F., \& Baron, E. 1999, ApJ, 512, 377

Herczeg, G. J., \& Hillenbrand, L. A. 2008, ApJ, 681, 594

Ingleby, L., Calvet, N., Herczeg, G., et al. 2013, ApJ, 767,112

Kenyon, S. J., \& Hartmann, L. 1995, ApJS, 101, 117

Koenig, X. P., Leisawitz, D. T., Benford, D. J., et al. 2012, ApJ, 744, 130

Königl, A. 1991, ApJ, 370, L39

Lee, H.-T., \& Chen, W. P., 2007, ApJ, 657, 884

Lin, D. N. C., \& Papaloizou, J. C. B. 1993: in Protostars and planets III, eds. E. H. Levy, \& J. I. Lunine (University of Arizona Press), 749

Looper, D., Mohanty, S., Bochanski, J. J., et al. 2010, ApJ, 714, 46

Lynds, B. T. 1962, ApJS, 7, 1

Manara, C. F., Testi, L., Rigliaco, E., et al. 2013, A\&A, 551, A107

Meyer, M. R., Calvet, N., \& Hillenbrand, L. A. 1997, AJ, 114, 288

Modigliani, A., Mulas, G., Porceddu, I., et al. 2004, The Messenger, 118, 8

Mohanty, S., Jayawardhana, R., \& Basri, G. 2005, ApJ, 626, 498

Muzerolle, J., Hartmann, L., \& Calvet, N. 1998, AJ, 116, 2965

Muzerolle, J., Hillenbrand, L., Calvet, N., Briceño, C., Hartmann, L. 2003, ApJ, 592,266

Muzerolle, J., Luhman, K., Briceño, C., et al. 2005, ApJ, 625, 906

Natta, A., Testi, L., \& Randich, S. 2006, A\&A, 452, 245

Nguyen, D. C., Scholz, A., van Kerkwijk, M. H., Jayawardhana, R., \& Brandeker, A. 2009, ApJ, 694, L153

Palla, F., Stahler, S. W. 1999, ApJ, 525, 772

Palla, F., Randich, S., Pavlenko, Y. V., Flaccomio, E., \& Pallavicini, R. 2007, ApJ, 659, 41

Pavlenko, Y. V., \& Magazzù, A. 1996, A\&A, 311, 961

Ribas, A., Merín, B., Bouy, H., \& Maud, L. T. 2014, A\&A, 561, A54

Rigliaco, E., Natta, A., Randich, S., Testi, L., \& Biazzo, K. 2011a, A\&A, 525 A47

Rigliaco, E., Natta, A., Randich, S., et al. 2011b, A\&A, 526, A6

Rigliaco, E., Natta, A., Testi, L., et al. 2012, A\&A, 548, A56

Schisano, E., Covino, E., Alcalá, et al. 2009, A\&A, 501, 1013

Sergison, D. J., Mayne, N. J., Naylor, R., Jeffries, R. D., \& Bell, P. M. 2013, MNRAS, 434, 966

Shu, F., Najita, J., Ostriker, E., \& Wilkin, F. 1994, ApJ, 429, 781

Stanke, R., Smith, M. D., Gredel, R., \& Szokoly, G. 2002, ApJ, 393, 251

Tonry, J., \& Davis, M. 1979, ApJ, 84, 1511

Uchida, Y., \& Shibata, K. 1985, PASJ, 37, 515

Vorovyov, E. J., \& Basu, S. 2008, ApJ, 676, 139

White, R. J., \& Basri, G. 2003, ApJ, 582, 1109

White, R. J., \& Hillenbrand, L. A. 2004, ApJ, 616, 998

Pages 9 to 16 are available in the electronic edition of the journal at http://wWw . aanda.org 
K. Biazzo et al.: On the accretion properties of young stellar objects in the L1615/L1616 cometary cloud

Table 3. Observing log, radial velocity, and lithium content for the stars observed with FLAMES.

\begin{tabular}{|c|c|c|c|c|c|c|c|}
\hline ID name & $\begin{array}{c}\text { JD } \\
(+2450000)\end{array}$ & Instrument & $\begin{array}{c}\# \\
\text { obs. }\end{array}$ & $\begin{array}{c}V_{\text {rad }} \\
\left(\mathrm{km} \mathrm{s}^{-1}\right)\end{array}$ & $\begin{array}{l}E W_{\mathrm{Li}} \\
(\mathrm{m} \AA)\end{array}$ & $\begin{array}{l}\log n(\mathrm{Li}) \\
\quad(\mathrm{dex})\end{array}$ & Comment \\
\hline \multirow[t]{2}{*}{ TTS J050646.1-031922 } & 3797.0722 & GIRAFFE & 2 & $26.2 \pm 6.6$ & 587 & 3.70 & $\mathrm{~N} 1, \mathrm{~N} 2 ; \mathrm{S} 1, \mathrm{~S} 2$ \\
\hline & 3797.1097 & & & $25.9 \pm 6.9$ & 594 & & $\mathrm{~N} 1, \mathrm{~N} 2 ; \mathrm{S} 1, \mathrm{~S} 2$ \\
\hline \multirow[t]{3}{*}{ RX J0506.8-0318 } & 3795.0535 & GIRAFFE & 3 & $21.6 \pm 0.7$ & 604 & 3.15 & \\
\hline & 3795.1009 & " & & $21.7 \pm 2.7$ & 605 & & \\
\hline & 3796.0344 & " & & $22.6 \pm 0.3$ & 581 & & \\
\hline \multirow[t]{3}{*}{ TTS J050647.5-031910 } & 3795.0535 & GIRAFFE & 3 & $\ldots$ & $\ldots$ & $\ldots$ & LSN \\
\hline & 3795.1009 & & & $28.6 \pm 5.3$ & $\ldots$ & & \\
\hline & 3796.0344 & $"$ & & & & & LSN \\
\hline \multirow[t]{2}{*}{ RX J0506.8-0327 } & 3795.0535 & GIRAFFE & 2 & $26.3 \pm 2.3$ & 566 & 3.50 & \\
\hline & 3795.1009 & $"$ & & $26.8 \pm 1.8$ & 550 & & \\
\hline RX J0506.8-0305 & 3796.0344 & GIRAFFE & 1 & $19.1 \pm 4.0$ & 565 & 3.35 & \\
\hline \multirow[t]{2}{*}{ TTS J050649.8-032104 } & 3795.1009 & GIRAFFE & 2 & $\ldots$ & $\ldots$ & & LSN \\
\hline & 3796.0344 & & & $\ldots$ & & & LSN \\
\hline RX J0506.9-0319 NW & 3795.1009 & GIRAFFE & 1 & $21.3 \pm 2.2$ & 412 & 2.70 & \\
\hline \multirow[t]{2}{*}{ RX J0506.9-0319 SE } & 3797.0722 & GIRAFFE & 2 & $27.4 \pm 5.8$ & 565 & 3.40 & $\mathrm{~N} 1, \mathrm{~N} 2 ; \mathrm{S} 1, \mathrm{~S} 2$ \\
\hline & 3797.1097 & & & $27.2 \pm 4.7$ & 601 & & $\mathrm{~N} 1, \mathrm{~N} 2 ; \mathrm{S} 1, \mathrm{~S} 2$ \\
\hline RX J0506.9-0320 W & 3795.0535 & GIRAFFE & 1 & $21.4 \pm 1.6$ & 562 & 3.35 & \\
\hline \multirow{4}{*}{ RX J0506.9-0320 E } & 3796.0344 & GIRAFFE & 4 & $22.9 \pm 3.3$ & 409 & 3.70 & \\
\hline & 3797.0298 & & & $23.5 \pm 3.3$ & 444 & & \\
\hline & 3797.0722 & $"$ & & $24.3 \pm 4.4$ & 385 & & \\
\hline & 3797.1096 & $"$ & & $23.9 \pm 5.6$ & 377 & & \\
\hline \multirow[t]{7}{*}{$\mathrm{LkH} \alpha 333$} & 3796.0344 & GIRAFFE & 1 & $25.9 \pm 1.6$ & 447 & 3.50 & \\
\hline & 3795.0535 & UVES & 6 & $27.0 \pm 0.5$ & 508 & & $\mathrm{O} 1, \mathrm{O} 2 ; \mathrm{T}$ \\
\hline & 3795.1009 & & & $\ldots$ & $\ldots$ & & LSN \\
\hline & 3796.0767 & " & & $27.8 \pm 0.5$ & 462 & & $\mathrm{O} 1, \mathrm{O} 2$ \\
\hline & 3797.0298 & " & & $28.4 \pm 0.6$ & 455 & & $\mathrm{O} 1, \mathrm{O} 2$ \\
\hline & 3797.1097 & " & & $28.2 \pm 2.0$ & 437 & & $\mathrm{O} 1, \mathrm{O} 2$ \\
\hline & 3797.0723 & " & & $27.9 \pm 1.1$ & 455 & & $\mathrm{O} 1, \mathrm{O} 2$ \\
\hline \multirow[t]{4}{*}{ L1616 MIR 4} & 3795.1009 & GIRAFFE & 4 & $24.3^{a}$ & 365 & 3.50 & \\
\hline & 3797.0298 & " & & $29.4 \pm 7.9$ & $\ldots$ & & LSN \\
\hline & 3797.0722 & $"$ & & $28.1^{a}$ & $\ldots$ & & LSN \\
\hline & 3797.1097 & $"$ & & $\ldots$ & $\ldots$ & & LSN \\
\hline \multirow[t]{3}{*}{ RX J0507.0-0318 } & 3795.0535 & GIRAFFE & 3 & $23.1 \pm 2.7$ & 556 & $>3.50$ & \\
\hline & 3795.1009 & & & $23.7 \pm 1.9$ & 583 & & $\mathrm{~T}$ \\
\hline & 3796.0344 & " & & $23.6 \pm 1.7$ & 601 & & \\
\hline \multirow[t]{2}{*}{ RX J0507.1-0321 } & 3795.0535 & GIRAFFE & 2 & $20.9 \pm 1.1$ & 575 & $>3.50$ & \\
\hline & 3796.0344 & & & $21.9 \pm 1.8$ & 550 & & \\
\hline RX J0507.2-0323 & 3796.0767 & GIRAFFE & 1 & $24.8 \pm 1.7$ & 525 & 3.40 & $\mathrm{~T}$ \\
\hline \multirow[t]{2}{*}{ RX J0507.3-0326 } & 3795.0535 & GIRAFFE & 2 & $21.4 \pm 1.1$ & 549 & 3.40 & \\
\hline & 3796.0767 & & & $21.8 \pm 1.8$ & 545 & & \\
\hline \multirow[t]{2}{*}{ TTS J050717.9-032433 } & 3795.0535 & GIRAFFE & 2 & $20.2^{a}$ & 524 & 3.50 & \\
\hline & 3796.0767 & & & $20.4^{a}$ & 530 & & \\
\hline \multirow[t]{3}{*}{ RX J0507.4-0320 } & 3795.0535 & GIRAFFE & 3 & $20.3^{a}$ & 587 & 3.50 & \\
\hline & 3796.0767 & " & & $19.8^{a}$ & 518 & & \\
\hline & 3796.0344 & $"$ & & $19.9^{a}$ & 608 & & \\
\hline \multirow[t]{3}{*}{ RX J0507.4-0317 } & 3795.0535 & GIRAFFE & 3 & $19.8 \pm 6.4$ & 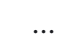 & 3.20 & LSN \\
\hline & 3796.0767 & & & $19.2^{a}$ & 457 & & \\
\hline & 3796.0344 & $"$ & & $20.0^{a}$ & 503 & & \\
\hline TTS J050729.8-031705 & 3795.0535 & GIRAFFE & 3 & $\ldots$ & $\ldots$ & 3.00 & LSN \\
\hline & 3796.0767 & $"$ & & $\ldots$ & & & LSN \\
\hline & 3796.0344 & $"$ & & $\ldots$ & 580 & & \\
\hline TTS J050730.9-031846 & 3795.0535 & GIRAFFE & 2 & $\ldots$ & $\ldots$ & $\ldots$ & LSN \\
\hline & 3796.0344 & & & $\ldots$ & $\ldots$ & & LSN \\
\hline TTS J050734.8-031521 & 3795.0535 & GIRAFFE & 3 & $\ldots$ & $\ldots$ & $\ldots$ & LSN \\
\hline & 3796.0767 & 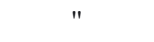 & & $\ldots$ & $\ldots$ & & LSN \\
\hline & 3796.0344 & & & & & & LSN \\
\hline RX J0507.6-0318 & 3795.0535 & GIRAFFE & 3 & $20.3 \pm 2.7$ & 567 & 2.75 & \\
\hline & 3796.0767 & $"$ & & $19.2 \pm 1.7$ & 524 & & \\
\hline & 3796.0344 & $"$ & & $19.5 \pm 4.1$ & 544 & & \\
\hline
\end{tabular}

Notes. ${ }^{(a)}$ Due to low S/N, few lines, late spectral type, and/or short wavelength coverage, the radial velocity error may be up to $60 \%$. LSN: low $\mathrm{S} / \mathrm{N}$ spectrum; T: template spectrum used for the CCF analysis. Some optical forbidden lines are observed in emission: $\mathrm{O} 1=[\mathrm{OI}] \lambda 6300.8 \AA$, $\mathrm{O} 2=[\mathrm{O} \mathrm{I}] \lambda 6363.8 \AA, \mathrm{S} 1=[\mathrm{S}$ II $] \lambda 6715.8 \AA, \mathrm{S} 2=[\mathrm{S} \mathrm{II}] \lambda 6729.8 \AA, \mathrm{N} 1=[\mathrm{N} \mathrm{II}] \lambda 6548.4 \AA, \mathrm{N} 2=[\mathrm{N}$ II $] \lambda 6583.4 \AA$. 
Table 4. Near-IR and mid-IR photometric data.

\begin{tabular}{|c|c|c|c|c|c|c|c|}
\hline ID name & $\begin{array}{c}J \\
\text { (mag) } \\
\end{array}$ & $\begin{array}{c}\text { 2MASS } \\
H \\
(\mathrm{mag}) \\
\end{array}$ & $\begin{array}{c}K \mathrm{~s} \\
(\mathrm{mag})\end{array}$ & $\begin{array}{c}{[3.4]} \\
(\mathrm{mag}) \\
\end{array}$ & $\begin{array}{l}\text { WISE } \\
{[4.6]} \\
(\mathrm{mag})\end{array}$ & $\begin{array}{l}{[12.0]} \\
(\mathrm{mag}) \\
\end{array}$ & $\begin{array}{l}{[22.0]} \\
(\mathrm{mag}) \\
\end{array}$ \\
\hline 1RXS J045912.4-033711 & $.070 \pm 0.022$ & $9.616 \pm 0.024$ & $9.474 \pm 0.020$ & $9.386 \pm 0.023$ & $395 \pm 0.018$ & $9.299 \pm 0.033$ & $8.691^{b}$ \\
\hline 1RXS J050416.9-021426 & $10.661 \pm 0.024$ & $10.105 \pm 0.024$ & $9.984 \pm 0.025$ & $9.910 \pm 0.023$ & $9.936 \pm 0.020$ & $9.812 \pm 0.048$ & $8.943^{b}$ \\
\hline TTS J050513.5-034248 & $15.120 \pm 0.041$ & $14.598 \pm 0.053$ & $14.198 \pm 0.065$ & $14.011 \pm 0.030$ & $13.837 \pm 0.044$ & $12.041^{b}$ & $8.769^{b}$ \\
\hline TTS J050538.9-032626 & $13.287 \pm 0.024$ & $12.601 \pm 0.022$ & $12.387 \pm 0.024$ & $12.196 \pm 0.024$ & $12.093 \pm 0.023$ & $11.186 \pm 0.159$ & $7.745 \pm 0.152$ \\
\hline RX J0506.6-0337 & $10.657 \pm 0.022$ & $10.304 \pm 0.027$ & $10.205 \pm 0.023$ & $10.130 \pm 0.024$ & $10.138 \pm 0.021$ & $10.226 \pm 0.070$ & $9.067^{b}$ \\
\hline TTS J050644.4-032913 & $13.641 \pm 0.028$ & $13.038 \pm 0.031$ & $12.779 \pm 0.032$ & $12.613 \pm 0.025$ & $12.421 \pm 0.025$ & $11.411 \pm 0.146$ & $8.731 \pm 0.336$ \\
\hline TTS J050646.1-031922 & $13.218 \pm 0.025$ & $11.506 \pm 0.025$ & $10.322 \pm 0.023$ & $8.866 \pm 0.023$ & $8.148 \pm 0.018$ & $5.976 \pm 0.014$ & $3.918 \pm 0.026$ \\
\hline RX J0506.8-0318 & $11.849 \pm 0.023$ & $11.108 \pm 0.025$ & $10.955 \pm 0.023$ & $10.835 \pm 0.023$ & $10.819 \pm 0.020$ & $10.422^{b}$ & $7.696^{b}$ \\
\hline TTS J050647.5-031910 & $14.286 \pm 0.030$ & $13.410 \pm 0.030$ & $12.999 \pm 0.023$ & $12.586 \pm 0.024$ & $12.106 \pm 0.023$ & $9.229 \pm 0.180$ & $5.786 \pm 0.091$ \\
\hline RX J0506.8-C & $11.599 \pm 0.023$ & $10.855 \pm 0.027$ & $10.595 \pm$ & 10 & $10.430 \pm 0.020$ & $9.877 \pm 0.047$ & 8.337 \\
\hline RX J0506.8- & $12.939 \pm 0.021$ & $12.281 \pm 0.025$ & $12.058 \pm$ & 11 & $11.762 \pm 0.023$ & $11.868 \pm 0.305$ & $9.073^{b}$ \\
\hline TTS J05064 & $12.391 \pm 0.022$ & $11.526 \pm 0.027$ & $11.175 \pm$ & $47 \pm$ & $10.362 \pm 0.020$ & $7.215 \pm 0.020$ & $4.214 \pm 0.037$ \\
\hline TTS & $12.699 \pm$ & $11.346 \pm 0.026$ & $10.452 \pm$ & $9.845 \pm 0.023$ & $9.071 \pm 0.021$ & $5.756 \pm 0.016$ & $2.787 \pm$ \\
\hline TTS & $13.663 \pm$ & $12.971 \pm$ & $12.541 \pm$ & $\ldots$ & $\ldots$ & $\ldots$ & $\ldots$ \\
\hline TTS & $13.642 \pm$ & $12.984 \pm$ & 12.5 & $\ldots$ & $\ldots$ & $\ldots$ & $\ldots$ \\
\hline $\mathrm{RX}$ & $a$ & $11.939 \pm$ & & $\ldots$ & $\ldots$ & $\ldots$ & \\
\hline RX & $9^{a}$ & $10.050 \pm$ & & $\ldots$ & $\ldots$ & $\ldots$ & \\
\hline $\mathrm{RX}$ & $10.042 \pm 0.040$ & $8.889 \pm 0.042$ & 3. & $\ldots$ & $\ldots$ & $\ldots$ & \\
\hline $\mathrm{RX} \mathrm{J}$ & $10.366 \pm$ & $8.965 \pm$ & 8.0 & & . & . & \\
\hline 32046 & $15.984 \pm$ & $14.878 \pm$ & 14. & & & $\ldots$ & $\ldots$ \\
\hline $\mathrm{LkH} \alpha 333$ & $10.343 \pm 0.022$ & $9.239 \pm 0.024$ & $8.443 \pm 0.025$ & $6.981 \pm 0.030$ & $6.196 \pm 0.021$ & $.265 \pm 0.013$ & $1.888 \pm 0.018$ \\
\hline L1616 I & $13.010 \pm$ & $11.003 \pm 0.035$ & $9.524=$ & & & & \\
\hline RX J0507.0-0318 & $11.190 \pm 0.022$ & $10.344 \pm 0.027$ & $10.036 \pm 0.023$ & \pm 0.024 & $9.937 \pm 0.020$ & $9.103 \pm 0.051$ & $6.687 \pm 0.099$ \\
\hline TTS J050657.0-031640 & $13.040 \pm 0.023$ & $12.414 \pm 0.025$ & $12.062 \pm 0.023$ & $11.781 \pm 0.024$ & $11.309 \pm 0.022$ & $8.964 \pm 0.052$ & $6.820 \pm 0.092$ \\
\hline TTS J050704.7-030241 & $14.809 \pm 0.036$ & $14.208 \pm 0.049$ & $13.964 \pm$ & $13.795 \pm 0.029$ & $13.569 \pm 0.037$ & $12.652^{b}$ & $9.104^{b}$ \\
\hline TTS J050705.3-030006 & $12.517 \pm 0.023$ & $11.789 \pm 0.027$ & $11.614 \pm$ & $11.594 \pm 0$. & $11.539 \pm 0.023$ & $11.919^{b}$ & $8.931^{b}$ \\
\hline RX J0507.1-0321 & $12.153 \pm 0.025$ & $11.357 \pm 0.027$ & $10.926 \pm 0.022$ & $10.656 \pm 0.023$ & $10.104 \pm 0.022$ & $7.787 \pm 0.021$ & $5.530 \pm 0.043$ \\
\hline-031703 & $14.660 \pm 0.033$ & $14.067 \pm 0.041$ & $13.820 \pm$ & $98 \pm 0.027$ & $13.163 \pm 0.032$ & $10.891 \pm 0.145$ & $8.390 \pm$ \\
\hline RX JC & $11.659 \pm 0.020$ & $11.053 \pm 0.025$ & $10.911 \pm$ & $10.806 \pm 0.022$ & $10.799 \pm 0.021$ & $10.012 \pm 0.060$ & $7.505 \pm 0.160$ \\
\hline 031722 & $13.918 \pm 0.031$ & $12.855 \pm 0.030$ & $12.100 \pm 0.030$ & $\ldots$ & $\ldots$ & $\ldots$ & $\ldots$ \\
\hline RX J0507.3-0326 & $11.454 \pm 0$. & $10.780 \pm 0.024$ & $10.628 \pm 0.022$ & $08 \pm$ & $78 \pm 0.019$ & $9 \pm$ & $8.989^{b}$ \\
\hline TTS J050717.9-032433 & $13.099 \pm 0.0$ & $12.426 \pm 0.028$ & $12.178 \pm 0.028$ & & $12.023 \pm 0.023$ & $69 \pm$ & $9.182^{b}$ \\
\hline RX J0507.4-0320 & $12.305 \pm 0.024$ & $11.716 \pm 0.025$ & $11.440 \pm 0.023$ & $11.305 \pm 0.023$ & $11.127 \pm 0.022$ & $11.021 \pm 0$. & $8.968^{b}$ \\
\hline $07.4-0317$ & $13.173 \pm 0.026$ & $12.469 \pm 0.027$ & $12.254 \pm 0.029$ & $12.161 \pm 0.023$ & $12.019 \pm 0.024$ & $11.943 \pm 0.285$ & $8.956^{b}$ \\
\hline TTS J050729.8-031705 & $14.937 \pm 0.035$ & $14.230 \pm 0.040$ & $13.964 \pm 0.053$ & $13.821 \pm 0.029$ & $13.545 \pm 0.038$ & $12.570^{b}$ & $8.995^{b}$ \\
\hline TTS J050730.9-031846 & $16.379 \pm 0.104$ & $15.954 \pm 0.178$ & $15.294 \pm 0.157$ & $14.830 \pm 0.038$ & $14.113 \pm 0.051$ & $12.272^{b}$ & $9.153^{b}$ \\
\hline TTS J050733.6-032517 & $14.707 \pm 0.040$ & $14.138 \pm 0.044$ & $13.839 \pm 0.055$ & $13.667 \pm 0.028$ & $13.470 \pm 0.036$ & $12.457^{b}$ & $9.030^{b}$ \\
\hline TTS J050734.8-031521 & $14.430 \pm 0.030$ & $13.820 \pm 0.030$ & $13.515 \pm 0.045$ & $13.396 \pm 0.026$ & $13.163 \pm 0.032$ & $12.397^{b}$ & $8.930^{b}$ \\
\hline RX J0507.6-0318 & $12.288 \pm 0.021$ & $11.616 \pm 0.025$ & $11.461 \pm 0.025$ & $11.333 \pm 0.023$ & $11.322 \pm 0.022$ & $11.959 \pm 0.346$ & $8.693^{b}$ \\
\hline TTS J050741.0-032253 & $13.517 \pm 0.026$ & $12.809 \pm 0.028$ & $12.572 \pm 0.034$ & $12.520 \pm 0.023$ & $12.377 \pm 0.026$ & $12.171^{b}$ & $8.903^{b}$ \\
\hline TTS J050741.4-031507 & $13.506 \pm 0.026$ & $12.886 \pm 0.030$ & $12.638 \pm 0.029$ & $12.456 \pm 0.024$ & $12.263 \pm 0.025$ & $12.115^{b}$ & $9.039^{b}$ \\
\hline TTS J050752.0-032003 & $14.521 \pm 0.033$ & $13.979 \pm 0.038$ & $13.511 \pm 0.037$ & $13.326 \pm 0.027$ & $12.920 \pm 0.030$ & $11.811 \pm 0.258$ & $8.768^{b}$ \\
\hline TTS J050801.4-032255 & $12.511 \pm 0.022$ & $11.628 \pm 0.024$ & $11.231 \pm 0.023$ & $10.683 \pm 0.023$ & $10.143 \pm 0.021$ & $7.022 \pm 0.016$ & $4.729 \pm 0.028$ \\
\hline TTS J050801.9-031732 & $12.392 \pm 0.024$ & $11.663 \pm 0.023$ & $11.327 \pm 0.025$ & $10.624 \pm 0.024$ & $10.268 \pm 0.021$ & $9.397 \pm 0.038$ & $6.945 \pm 0.077$ \\
\hline TTS J050804.0-034052 & $12.996 \pm 0.027$ & $12.332 \pm 0.034$ & $12.071 \pm 0.024$ & $11.940 \pm 0.035$ & $11.824 \pm 0.029$ & $11.446 \pm 0.174$ & $8.561^{b}$ \\
\hline TTS J050836.6-030341 & $11.931 \pm 0.024$ & $11.159 \pm 0.025$ & $10.726 \pm 0.022$ & $10.153 \pm 0.023$ & $9.685 \pm 0.020$ & $8.104 \pm 0.019$ & $6.351 \pm 0.053$ \\
\hline TTS J050845.1-031653 & $12.370 \pm 0.035$ & $11.822 \pm 0.037$ & $11.462 \pm 0.032$ & $11.222 \pm 0.031$ & $11.033 \pm 0.031$ & $10.644 \pm 0.097$ & $8.848^{b}$ \\
\hline RX J0509.0-0315 & $9.914 \pm 0.024$ & $9.530 \pm 0.024$ & $9.408 \pm 0.021$ & $9.337 \pm 0.023$ & $9.360 \pm 0.019$ & $9.321 \pm 0.035$ & $8.706^{b}$ \\
\hline RX J0510.1-0427 & $9.684 \pm 0.024$ & $9.144 \pm 0.025$ & $8.991 \pm 0.021$ & $8.913 \pm 0.022$ & $8.930 \pm 0.022$ & $8.899 \pm 0.026$ & $8.800 \pm 0.364$ \\
\hline 1RXS J051011.5-025355 & $10.454 \pm 0.022$ & $9.952 \pm 0.024$ & $9.730 \pm 0.023$ & $9.794 \pm 0.023$ & $9.342 \pm 0.019$ & $5.371 \pm 0.016$ & $3.140 \pm 0.021$ \\
\hline RX J0510.3-0330 & $10.038^{a}$ & $9.806 \pm 0.060$ & $9.749 \pm 0.049$ & $9.177 \pm 0.022$ & $9.205 \pm 0.019$ & $9.283 \pm 0.032$ & $8.941 \pm 0.409$ \\
\hline 1RXS J051043.2-031627 & $10.079 \pm 0.026$ & $9.735 \pm 0.022$ & $9.648 \pm 0.025$ & $9.558 \pm 0.022$ & $9.584 \pm 0.020$ & $9.534 \pm 0.036$ & $8.555^{b}$ \\
\hline RX J0511.7-0348 & $10.342 \pm 0.026$ & $9.868 \pm 0.022$ & $9.806 \pm 0.023$ & $9.700 \pm 0.023$ & $9.702 \pm 0.019$ & $9.659 \pm 0.041$ & $8.537^{b}$ \\
\hline RX J0512.3-0255 & $10.425 \pm 0.023$ & $9.688 \pm 0.023$ & $9.140 \pm 0.019$ & $8.426 \pm 0.023$ & $8.043 \pm 0.020$ & $7.288 \pm 0.016$ & $4.479 \pm 0.027$ \\
\hline
\end{tabular}

Notes. ${ }^{(a)}$ Upper limit on magnitude. The source is not resolved in a consistent fashion with the other bands. ${ }^{(b)}$ This magnitude corresponds to upper limit $(S / N<2)$. 
K. Biazzo et al.: On the accretion properties of young stellar objects in the L1615/L1616 cometary cloud

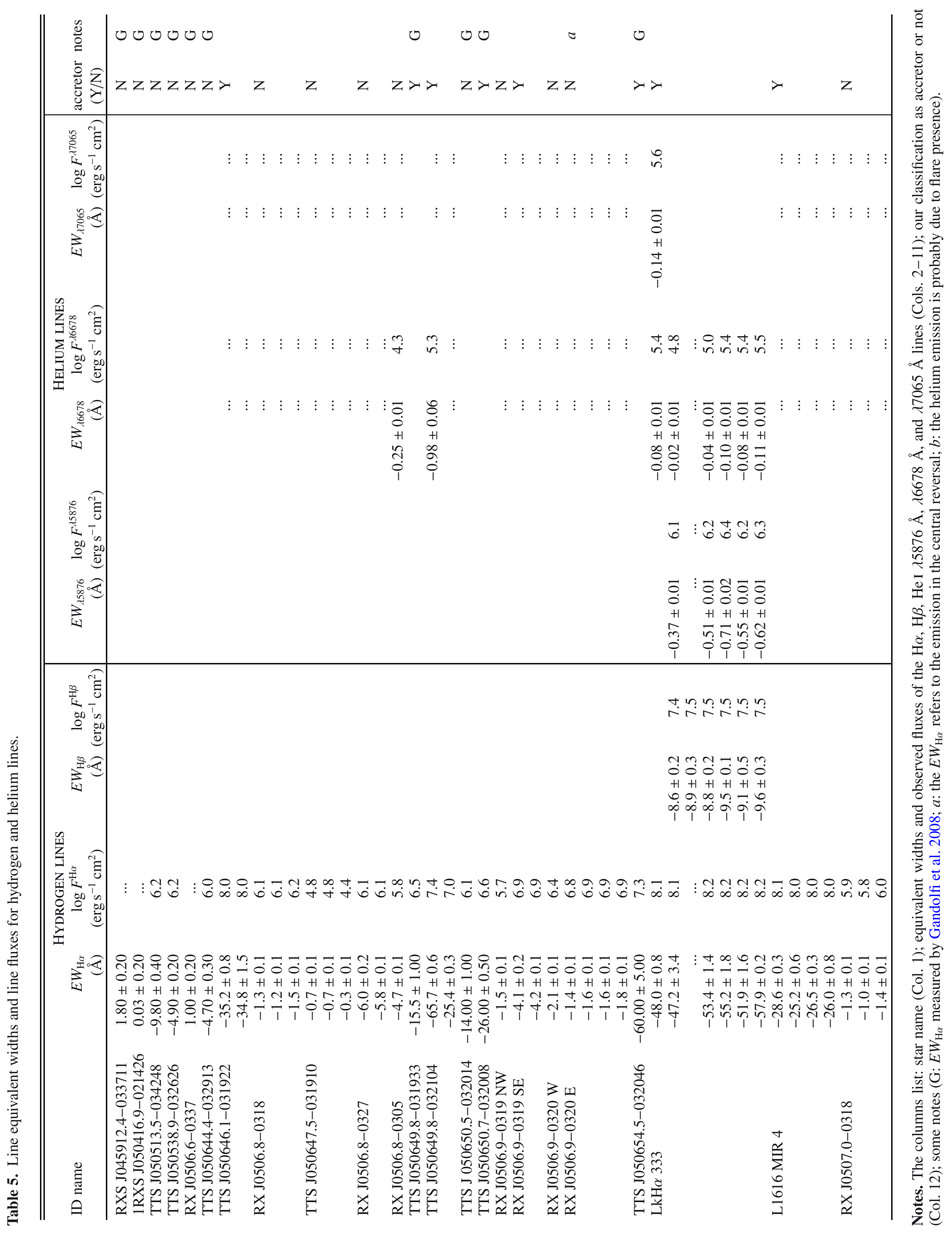




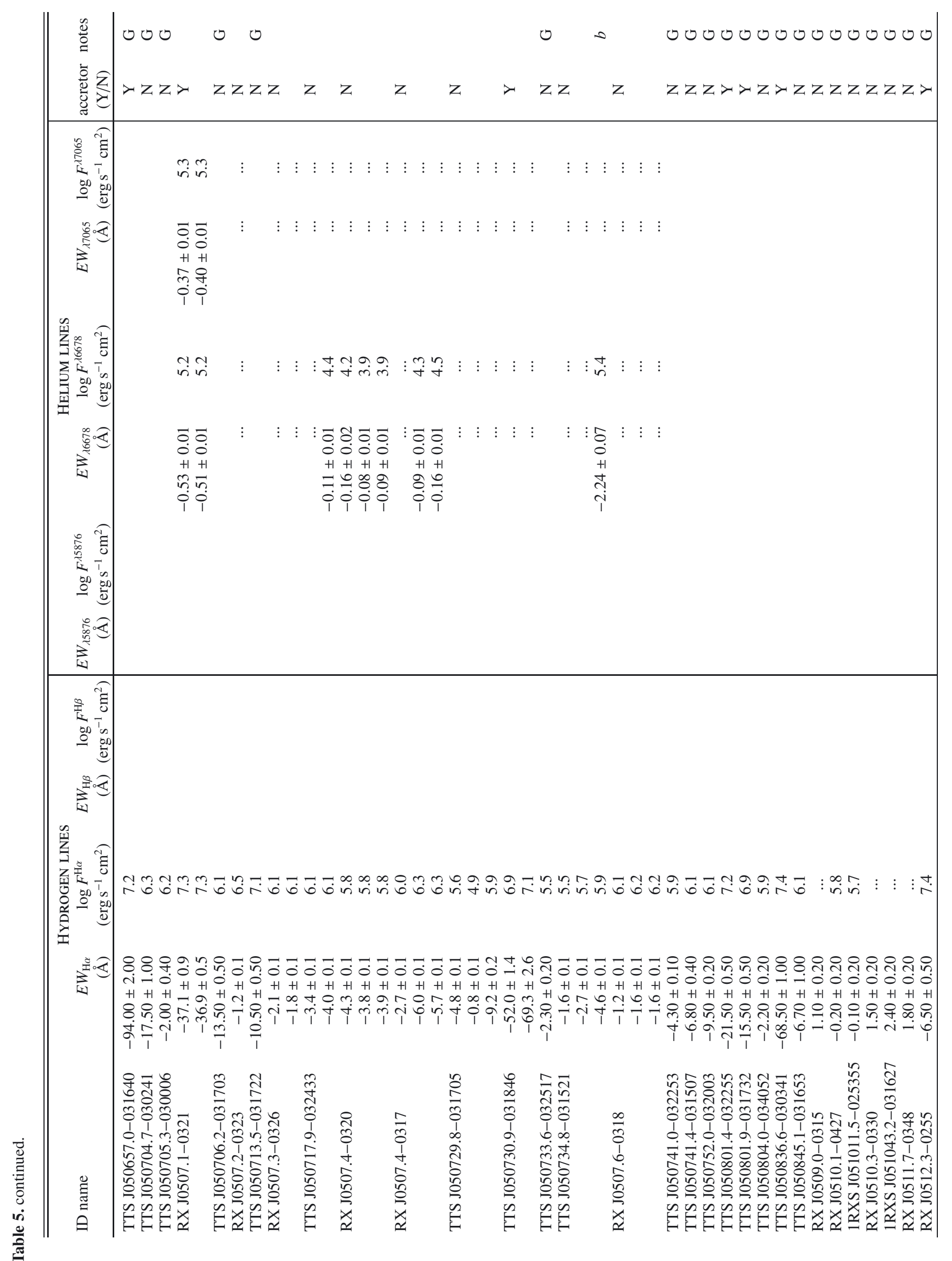


K. Biazzo et al.: On the accretion properties of young stellar objects in the L1615/L1616 cometary cloud

Table 6. Mean accretion luminosities, stellar masses, and mass accretion rates derived for the 15 accretors in L1615/L1616 from the Ba98+Ch00, DM97, and PS99 stellar masses.

\begin{tabular}{l|c|cc|cc|cc}
\hline \hline ID name & $\log \left\langle\frac{L_{\mathrm{acc}}}{L_{\odot}}\right\rangle$ & \multicolumn{2}{|c|}{$\mathrm{Ba} 98+\mathrm{Ch} 00$} & \multicolumn{2}{c|}{ DM97 } & \multicolumn{2}{c}{ PS99 } \\
& & $\begin{array}{c}M_{\star} \\
\left(M_{\odot}\right)\end{array}$ & $\begin{array}{l}\log \dot{M}_{\mathrm{acc}} \\
\left(M_{\odot} \mathrm{yr}^{-1}\right)\end{array}$ & $\begin{array}{c}M_{\star} \\
\left(M_{\odot}\right)\end{array}$ & $\begin{array}{l}\log \dot{M}_{\mathrm{acc}} \\
\left(M_{\odot} \mathrm{yr}^{-1}\right)\end{array}$ & $\begin{array}{c}M_{\star} \\
\left(M_{\odot}\right)\end{array}$ & $\begin{array}{c}\log \dot{M}_{\mathrm{acc}} \\
\left(M_{\odot} \mathrm{yr}^{-1}\right)\end{array}$ \\
\hline TTS J050646.1-031922 & -1.0 & $\ldots$ & $\ldots$ & 0.75 & -8.0 & 1.35 & -8.3 \\
TTS J050649.8-031933 & -2.6 & 0.47 & -9.4 & 0.22 & -9.1 & 0.25 & -9.1 \\
TTS J050649.8-032104 & -1.6 & 0.87 & -8.5 & 0.35 & -8.1 & 0.50 & -8.3 \\
TTS J050650.7-032008 & -2.9 & 0.26 & -9.6 & 0.19 & -9.5 & 0.18 & -9.5 \\
RX J0506.9-0319 SE & -1.9 & 1.40 & -9.0 & 0.45 & -8.5 & 0.93 & -8.8 \\
TTS J050654.5-032046 & -3.0 & 0.26 & -10.0 & 0.25 & -10.0 & 0.22 & -10.0 \\
LkH $\alpha$ 333 & -0.3 & $\ldots$ & $\ldots$ & 0.75 & -7.0 & 1.70 & -7.3 \\
L1616 MIR 4 & -0.7 & $\ldots$ & $\ldots$ & 1.70 & -7.9 & 1.75 & -8.0 \\
TTS J050657.0-031640 & -2.3 & 0.27 & -9.0 & 0.19 & -8.9 & 0.18 & -8.8 \\
RX J0507.1-0321 & -1.8 & 0.80 & -8.9 & 0.40 & -8.6 & 0.50 & -8.7 \\
TTS J050730.9-031846 & -3.8 & 0.11 & -10.7 & 0.15 & -10.9 & 0.13 & -10.8 \\
TTS J050801.4-032255 & -2.2 & 0.88 & -9.4 & 0.47 & -9.1 & 0.56 & -9.2 \\
TTS J050801.9-031732 & -2.5 & 0.80 & -9.6 & 0.44 & -9.4 & 0.50 & -9.4 \\
TTS J050836.6-030341 & -1.6 & 0.75 & -8.6 & 0.32 & -8.2 & 0.44 & -8.4 \\
RX J0512.3-0255 & -1.6 & $\ldots$ & $\ldots$ & 1.30 & -8.9 & 1.50 & -8.9 \\
\hline
\end{tabular}




\section{Appendix A: Radial velocity and lithium abundance} determinations

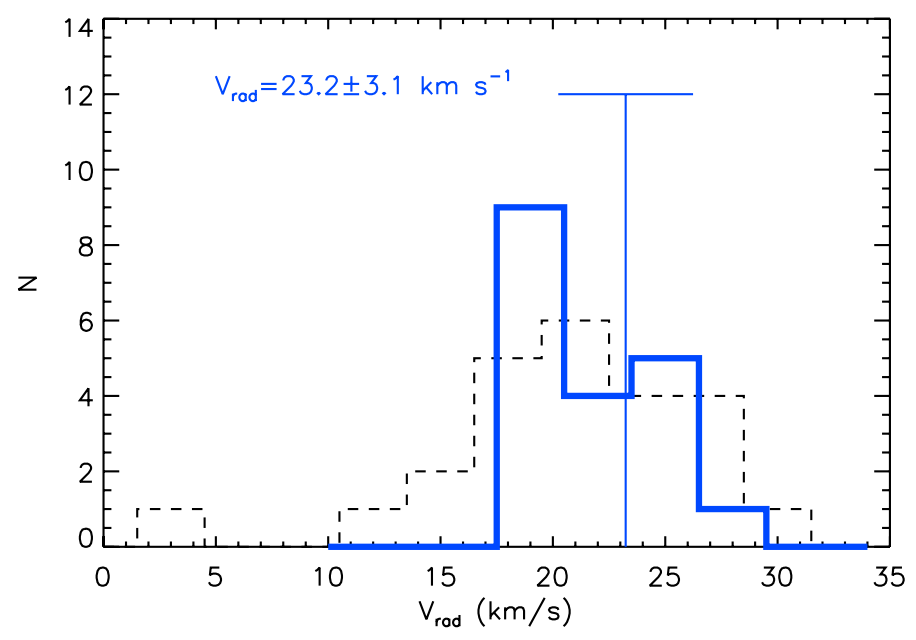

Fig. A.1. Average RV distribution of the L1615/L1616 low-mass stars (thick solid line). The bar indicates the mean RV value and its standard deviation from the average. The dashed line represents the distribution obtained by Alcalá et al. (2004) for 24 stars in L1616.

\section{A.1. Radial velocity}

We were able to measure radial velocities for 19 objects out of the 23 YSOs for which FLAMES spectroscopy was available. We followed the same procedure as in Biazzo et al. (2012). We determined heliocentric RVs of the targets through the task FXCOR within the IRAF package RV, which cross-correlates the target and template spectra, excluding regions affected by broad lines or prominent telluric features. As GIRAFFE templates, we used RX J0507.2-0323 and RX J0507.0-0318 for the earliesttype and latest-type stars, respectively, while as UVES template we considered the first spectrum acquired for $\mathrm{LkH} \alpha 333$. We measured the RV of each template using the IRAF task RVIDLINES inside the RV package. This task measures RVs from a line list. We used 40 lines for the UVES spectra and 10 lines for the GIRAFFE spectra, obtaining $V_{\text {rad }}=24.8 \pm$ $1.7 \mathrm{~km} \mathrm{~s}^{-1}$ for RX J0507.2-0323, $V_{\text {rad }}=23.7 \pm 1.9 \mathrm{~km} \mathrm{~s}^{-1}$ for RX J0507.0-0318, and $V_{\text {rad }}=27.0 \pm 0.5 \mathrm{~km} \mathrm{~s}^{-1}$ for LkH $\alpha 333$.

We determined the centroids of the cross-correlation function (CCF) peaks by adopting Gaussian fits, and we computed the RV errors by FXCOR according to the fitted peak height and the antisymmetric noise (see Tonry \& Davis 1979). The RV values derived for each spectrum are listed in Table 3 with the corresponding uncertainties.

In Fig. A.1, we show the L1615/L1616 distribution of the RV measurements obtained from both UVES and GIRAFFE spectra. When more than one spectrum was acquired, we computed the average RV for each object.

\section{A.2. Lithium abundance}

We measured lithium equivalent widths $\left(\mathrm{EW}_{\mathrm{Li}}\right)$ by direct integration or by Gaussian fit using the IRAF task SPLOT. We estimated errors in $\mathrm{EW}_{\mathrm{Li}}$ in the following way: $i$ ) when only one spectrum was available, we adopted the standard deviation of three $\mathrm{EW}_{\mathrm{Li}}$ measurements; $i$ ) when more than one spectrum was gathered, we adopted the standard deviation of the measurements on the different spectra. Typical errors in $\mathrm{EW}_{\mathrm{Li}}$ are of 1-20 m $\AA$. Our $\mathrm{EW}_{\mathrm{Li}}$ measurements are consistent with the values of Gandolfi et al. (2008) within $17 \mathrm{~m} \AA$ on average.

We estimated mean lithium abundances $(\log n(\mathrm{Li}))$ from the average $\mathrm{EW}_{\mathrm{Li}}$ listed in Table 3 and the effective temperature ( $\left.T_{\text {eff }}\right)$ from Gandolfi et al. (2008), using the LTE curves-ofgrowth reported by Pavlenko \& Magazzù (1996) for $T_{\mathrm{eff}}>$ $4000 \mathrm{~K}$, and by Palla et al. (2007) for $T_{\text {eff }}<4000 \mathrm{~K}$. We derived the $\log g$ values as explained in Sect. 3. The main source of error in $\log n(\mathrm{Li})$ comes from the uncertainty in $T_{\text {eff }}$, which is $\Delta T_{\text {eff }} \sim 100 \mathrm{~K}$ (Gandolfi et al. 2008). Taking this value and a mean error of $10 \mathrm{~m} \AA$ in $\mathrm{EW}_{\mathrm{Li}}$ into account, we estimated an uncertainty in $\log n(\mathrm{Li})$ ranging from $\sim 0.05-0.30$ dex for cooler stars $\left(T_{\text {eff }} \sim 3000 \mathrm{~K}\right)$ down to $\sim 0.05-0.15$ dex for warmer stars $\left(T_{\text {eff }} \sim 5000 \mathrm{~K}\right)$, depending on the $\mathrm{EW}_{\mathrm{Li}}$ value. Moreover, the $\log g$ value affects the lithium abundance, in the sense that the lower the surface gravity the higher the lithium abundance, and vice versa. In particular, the difference in $\log n(\mathrm{Li})$ may rise to $\sim \pm 0.1$ dex when considering stars with mean values of $\mathrm{EW}_{\mathrm{Li}}=$ $500 \mathrm{~m} \AA$ and $T_{\text {eff }}=4500 \mathrm{~K}$ and assuming $\Delta \log g=\mp 0.15 \mathrm{dex}$, which is the mean error derived from the Gandolfi et al. (2008) stellar parameters.

\section{Appendix B: $M_{\star}$ and $\dot{M}_{\text {acc }}$ as determined from different evolutionary tracks}

Gandolfi et al. (2008) have derived the mass of 56 members of L1615/L1616 by comparing the location of each object on the HR diagram with the theoretical PMS evolutionary tracks by Baraffe et al. (1998) and Chabrier et al. (2000) - Ba98+Ch00, D’Antona \& Mazzitelli (1997) - DM97, and Palla \& Stahler (1999) - PS99, which are available in the mass ranges $0.003 M_{\odot} \leq M_{\star} \leq 1.40 M_{\odot}, 0.017 M_{\odot} \leq M_{\star} \leq 3 M_{\odot}$, $0.1 M_{\odot} \leq M_{\star} \leq 6 M_{\odot}$, for Ba98+Ch00, DM97, and PS99 models, respectively. The usage of masses computed from different evolutionary tracks allowed us to estimate the model-dependent uncertainties in $\dot{M}_{\text {acc }}$ associated with the derived masses.

In Fig. B.1, we show the comparison between the masses derived from the three sets of tracks for the accreting objects. The largest residuals are seen when comparing the Ba98+Ch00 and DM97 tracks. Figure B.2 shows the comparisons of the mass accretion rates when calculated using the different evolutionary tracks. It can be seen that the $\log \dot{M}_{\text {acc }}$ values are rather independent of the choice of the PMS track. Therefore, it is the modeldependent uncertainty in mass that produces the dispersion in the $\log \dot{M}_{\text {acc }}$ vs. $\log M_{\star}$ plot, whereas the $\log \dot{M}_{\text {acc }}$ values are practically insensitive to the choice of the evolutionary model.

\section{Appendix C: Notes on individual objects}

\section{C.1. TTS 050730.9-031846: a subluminous YSO with edge-on disk?}

The star TTS 050730.9-031846 appears to be subluminous in the HR diagram reported by Gandolfi et al. (2008), in comparison with typical YSOs in L1615/L1616 of similar effective temperature. Subluminous YSOs have been found in other SFRs, like L1630N, L1641, Lupus, and Taurus (see Fang et al. 2009, 2013; Comerón et al. 2003; White \& Hillenbrand 2004; Looper et al. 2010; Alcalá et al. 2014). Among the hypotheses that explain the subluminosity of these YSOs are the following: $i$ ) these YSOs are believed to be embedded or to harbor flared disks with high inclination angles. In this case, the stellar photospheric light and any emission due to physical processes in the inner disk 
K. Biazzo et al.: On the accretion properties of young stellar objects in the L1615/L1616 cometary cloud
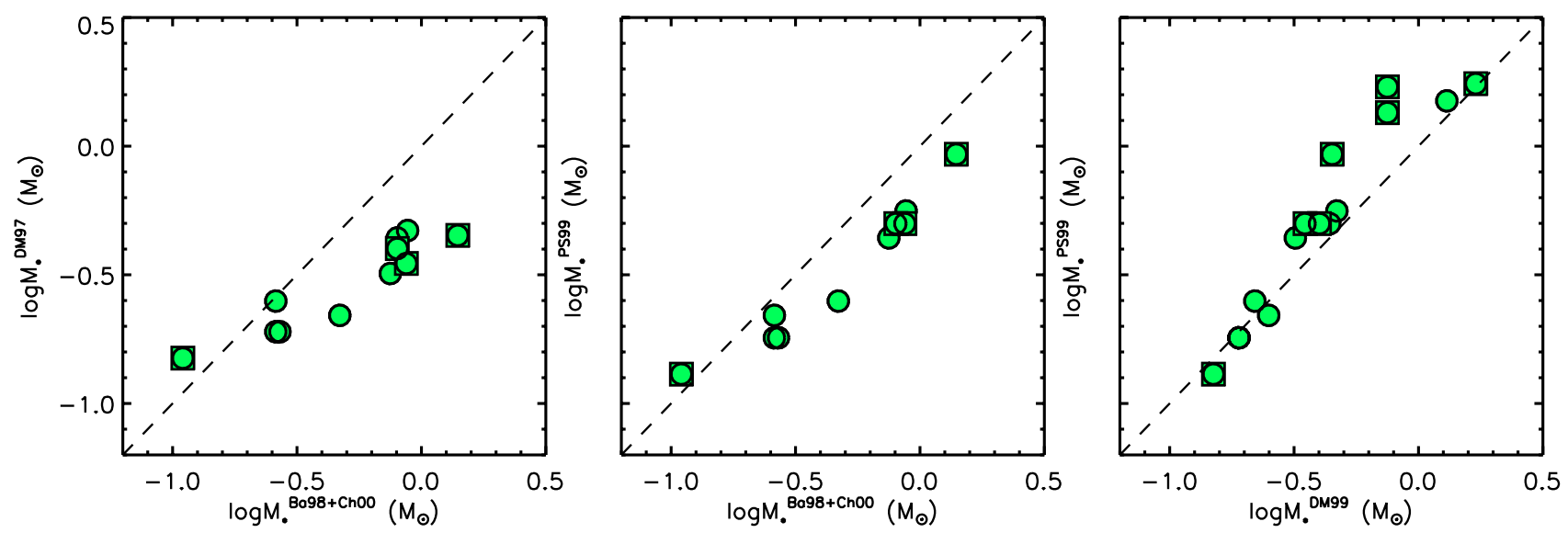

Fig. B.1. Comparison between masses derived from the Ba98+Ch00 and DM97 tracks (left panel), the Ba98+Ch00 and PS99 tracks (middle panel), and the DM97 and PS99 tracks (right panel) evolutionary tracks. Symbols are as in Fig. 2.
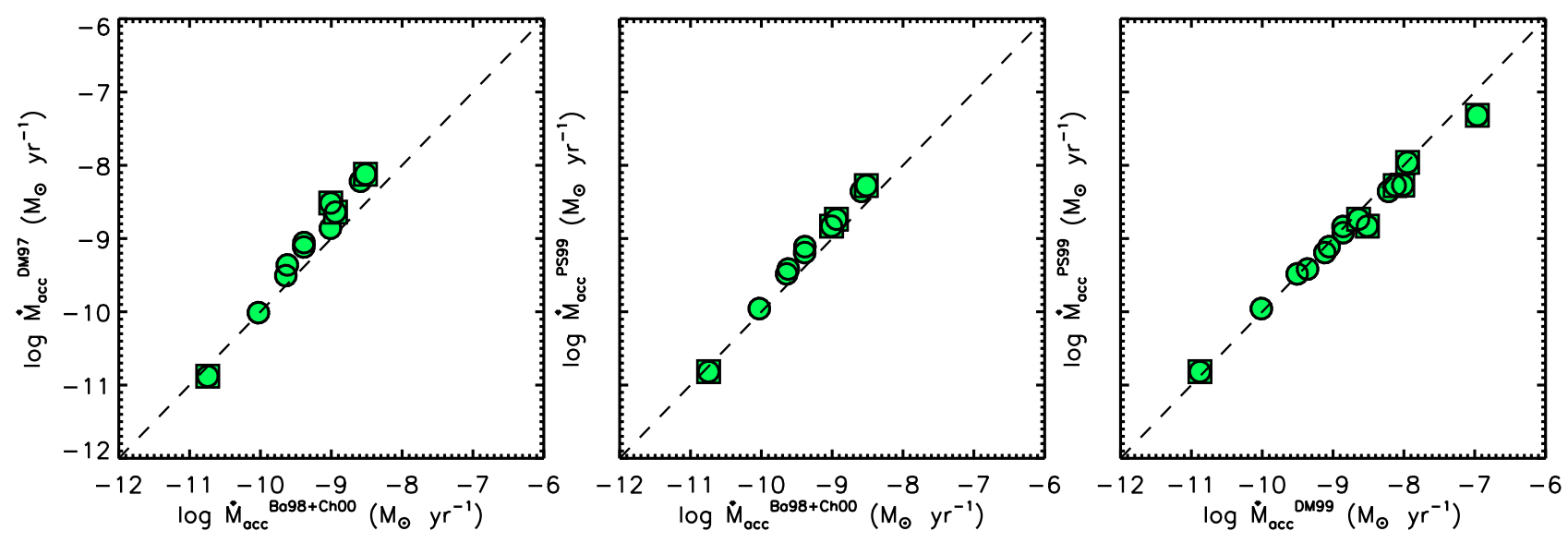

Fig. B.2. Comparison between mass accretion rates derived using the Ba98+Ch00 and DM97 tracks (left panel), the Ba98+Ch00 and PS99 tracks (middle panel), and the DM97 and PS99 tracks (right panel). Symbols are as in Fig. 2.

region are severely suppressed by the edge-on disk; $i$ ) other authors (Baraffe \& Chabrier 2010) argue that episodic strong accretion during the PMS evolution produces objects with smaller radius, higher central temperatures, and hence lower luminosity, compared to the nonaccreting counterparts of the same age and mass.

The anomalous position of TTS 050730.9-031846 on the $J-H$ versus $H-K$ s diagram (Fig. 2) would favor the hypothesis of a YSO with a high inclination angle in which both the stellar luminosity and the accretion luminosity are suppressed by the optically thick edge-on disk. A star with the effective temperature of TTS $050730.9-031846(\sim 3100 \mathrm{~K})$, at the age of L1615/L1616 ( 3 Myr; Gandolfi et al. 2008) should have a mass $M_{\star} \sim 0.1 M_{\odot}$ and should be a factor of $\sim 5$ more luminous than observed. This would imply that the mass accretion rate of the star should be a factor (5) ${ }^{1.5}$ higher than observed (Alcalá et al. 2014). This means values of $\sim 1.8,1.3,1.4 \times 10^{-10} M_{\odot} \mathrm{yr}^{-1}$ for the masses drawn from the Ba98+Ch00, DM97, PS99 tracks, respectively, i.e., similar to the $\dot{M}_{\text {acc }}$ measured in YSOs with the same mass (see Fig. 7).

An edge-on disk may also produce variable circumstellar extinction, inducing changes in the continuum that produce strong variations of the equivalent width of emission lines (see, e.g., the case of the transitional object $\mathrm{T}$ Chamaeleontis studied by Schisano et al. 2009). In fact, the $E W_{\mathrm{H} \alpha}=-290.00 \pm 30.00 \AA$ of TTS 050730.9-031846 measured by Gandolfi et al. (2008) is a factor of $\sim 6$ higher than what we measured here. Whether such strong variability is due to variable circumstellar extinction is not clear, but can only be confirmed by a simultaneous multiband photometric monitoring of the star.

\section{C.2. TTS $050649.8-031933$}

This star was classified as a WTTs by Gandolfi et al. (2008), but reanalyzing the three spectra acquired by the authors, helium and oxygen lines always appear in emission (as also reported in their Table 4). The results of our three measurements are $E W_{\mathrm{H} \alpha}=$ $-15.5,-21.7,-17.6 \AA$. Moreover, the WISE colors of the star are consistent with those of a Class II YSO. We thus classify TTS 050649.8-031933 as an accreting YSO.

\section{C.3. TTS 050649.8-032104 and L1616 MIR 4}

Besides the subluminous object discussed above, TTS 050649.8-032104 and L1616 MIR 4 are the other two YSOs in our sample with the strongest variations in the $\mathrm{H} \alpha$ line (see Fig. 1). In particular, for TTS 050649.8-032104 Gandolfi et al. (2008) measured $E W_{\mathrm{H} \alpha}=-195 \pm 5 \AA$, i.e., about three times higher than what we measure here, implying a difference of $\sim 0.6$ dex in $\log \dot{M}_{\text {acc }}$. In the case of L1616 MIR 4, the Gandolfi et al. (2008) result $\left(E W_{\mathrm{H} \alpha}=-60 \pm 6 \AA\right)$ is also 
about three times higher than our measurements, corresponding to a difference of $\sim 0.4 \mathrm{dex}$ in $\log \dot{M}_{\text {acc }}$.

\section{C.4. TTS $050713.5-031722$}

This star was classified as a CTTs by Gandolfi et al. (2008), but reanalyzing their spectrum (see their Table 4), neither helium nor oxygen lines appear in emission (as reported by the authors) and we measured an $\mathrm{H} \alpha$ equivalent width of $\sim-8 \AA$. Considering its spectral type (K8.5), it can be classified as nonaccretor according to the White \& Basri (2003) criteria.

\section{C.5. Other targets}

In some of the WISE/2MASS color-color diagrams of Fig. 3, the targets TTS 050647.5-031910, TTS 050706.2-031703, TTS 050752.0-032003, 1RXS J051011.5-025355, TTS 050734.8-031521, and TTS 050729.8-031705 appear to be close to or within the regions of Class II objects, according to Koenig et al. (2012). We indeed classified these six objects as nonaccretors (see Table 5), because their $\mathrm{H} \alpha$ equivalent widths and spectral types are consistent with this object class, according to the White \& Basri (2003) criteria. 